\title{
Les appareils orthodontiques invisibles et presque invisibles
}

\author{
Pascal BARON ${ }^{1,2 *}$ \\ 1 Pratique privée à Colomiers (Toulouse-France) \\ 2 Université de Toulouse III, 118 route de Narbonne, 31062 Toulouse Cedex 9, France
}

MOTS CLÉS :

Orthodontie linguale /

Aligneur transparent /

Appareil esthétique /

Attache linguale

KEYWORDS:

Lingual orthodontics /

Clear aligner /

Esthetic appliance /

Lingual bracket

\begin{abstract}
RÉSUMÉ - La demande des patients en appareil esthétique non visible est en permanente augmentation. Deux types d'orthodontie permettent d'obtenir un taux d'acceptabilité convenable auprès des patients. La technique linguale a évolué constamment en proposant des attaches pré-fabriquées ou des attaches sur mesure. Les possibilités techniques et les résultats ont été améliorés avec le temps. Les gouttières thermoplastiques sont une alternative aux dispositifs linguaux et sont en plein essor. Leurs propriétés spécifiques permettent de mieux apprécier leurs indications et leurs limites. Une revue de la littérature, l'évolution des systèmes et la présentation de quelques exemples cliniques de cas traités nous permettent de donner des éléments de compréhension de chacun de ces deux types d'appareil et de mettre en évidence les avantages et les inconvénients de chacun d'eux.
\end{abstract}

\begin{abstract}
Invisible and almost invisible orthodontic appliances. Patient demand for invisible esthetic orthodontic appliances is steadily increasing. Two types of orthodontic appliances have a high rate of patient acceptance. The lingual technique has continually evolved by offering both prefabricated brackets or custom-made brackets. The various techniques have been improved over time, and the results are better than ever. Thermoplastic aligners are an alternative to lingual devices and their use is rapidly growing. Their specific properties make it easier to assess their indications and limitations. A review of the literature, the development of these systems and a presentation of some clinical examples of treated cases will help us to provide some of the basics for understanding each of these two types of appliances and to demonstrate the advantages and disadvantages of each system.
\end{abstract}

\section{Introduction}

Depuis l'introduction de l'appareil Edgewise par Edouard H. Angle en 1928 [1, 2], l'ingénierie orthodontique s'est évertuée dans un premier temps à améliorer le concept initial jusqu'à en simplifier son usage. Radiographie standardisée, nouveaux matériaux, collage, arc droit sont, dans l'ordre chronologique, les avancées déterminantes qui ont permis un progrès technique constant tout au long du $20^{e}$ siècle grâce à l'apport concerté des cliniciens et des industriels. A partir des années 1980, s'est posé naturellement le problème de la visibilité des dispositifs orthodontiques. Le développement de l'orthodontie

* Auteur pour correspondance : baron.pascal@free.fr en faveur des adultes devenait incompatible avec des arcs et attaches métalliques vestibulaires. Aujourd'hui encore, ce facteur limitant est clairement objectivé par des études récentes. En 2009, l'institut de sondage Ipsos MORI ${ }^{1}$ révélait que $72 \%$ des personnes interrogées au Royaume-Uni ignorent l'existence d'appareils esthétiques. Parallèlement, la même année aux États-Unis, une enquête d'opinion donne un taux de satisfaction de $96 \%$ pour les patients traités par la technique Invisalign ${ }^{2}$, alors que trois américains sur quatre connaissent de nom cette marque commerciale.

\footnotetext{
${ }^{1}$ www.blos.co.uk/docs/BLOS_release_02-02-10.pdf

${ }^{2}$ www. aligntech.com/Pages/home.aspx
} 


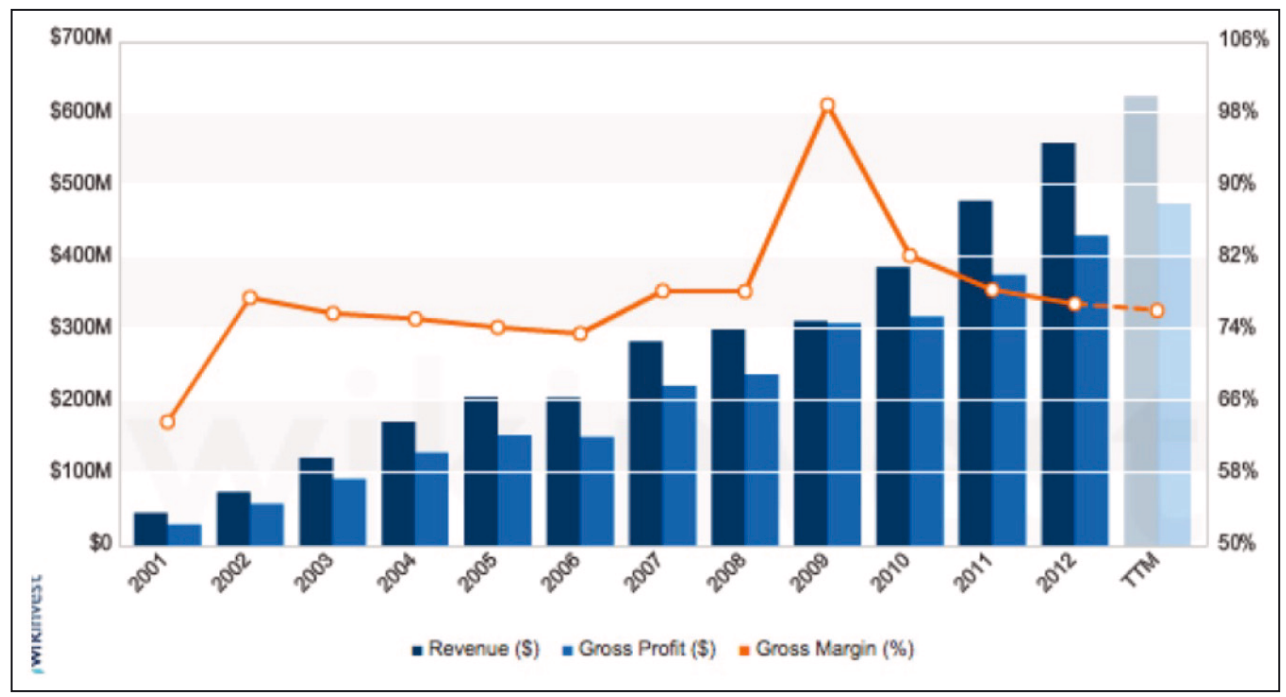

Figure 1

Progression des ventes d'Invisalign ${ }^{\circledR}$ depuis son introduction en bourse sur le NASDAQ.

Les premières évaluations indiquaient que $33 \%$ à $62 \%$ des adultes refusaient un traitement avec un appareil visible $[13,85]$. La recherche proprement dite sur l'acceptation esthétique des appareils orthodontiques commence avec les publications de Ziuchkovski, et al. [135] puis en 2008 Roswall, et al. [103]. Ils hiérarchisent les rapports d'acceptabilité qui passent de $90 \%$ à $55 \%$ dans l'ordre décroissant suivant : aligneurs et appareils linguaux, attaches céramiques, céramiques auto-ligaturants, appareils hybrides et métalliques, ces derniers étant jugés inacceptables pour plus de $42 \%$ des patients. Létude de Walton, et al. [125] suggère que l'acceptation et l'attractivité des appareils diffèrent entre les adolescents et les adultes. La tendance à préférer un appareil esthétique s'accroît avec l'âge, alors que les aligneurs transparents sont pour leur part plébiscités à tout âge. Jeremiah, et al. [64] soulignent, au-delà de l'acceptabilité de l'appareil, l'influence de celuici sur le jugement social qui se porte en faveur des dispositifs non visibles type lingual ou aligneurs.

\section{Historique}

Même si l'importance de l'aspect esthétique n'a été mesurée que récemment, les concepteurs cliniciens, conscients du problème, ont proposé des solutions cosmétiques dès la fin des années 1970. La première solution avancée est de placer les attaches sur la face linguale. Deux brevets sont déposés à l'US patent Office et à la Japanese patent Agency, respectivement par Craven Kurz et Kynia Fujita. Lorthodontie linguale est née mais rencontre un succès laborieux aussi bien en ce qui concerne la technique elle-même qu'en comptant le nombre de ses adeptes. Il faudra attendre plus de 25 ans avant que cette technique sorte de la confidentialité (cf. infra). Dans ce contexte, les brackets en céramique qui apparaissent en 1988 vont occuper pendant plus d'une décennie la place centrale de l'orthodontie esthétique. Invisalign (Align Technology, Santa Clara, CA) apparaît en 1999 aux États-Unis et un an plus tard en Europe avec, pour la première fois, un concept qui s'écarte radicalement des principes de base et qui se propose de déplacer les dents sans brackets ni arcs. En plus d'une nouvelle technique, il s'agit d'un nouvel opérateur dans le monde de l'orthodontie qui va s'adresser directement aux patients à grand renfort de campagnes publicitaires [36]. Il faudra cependant attendre plusieurs années et les effets de différentes stratégies commerciales avant d'envisager une diffusion planétaire qui aujourd'hui en 2013 occupe une part grandissante du marché de l'orthodontie ${ }^{3}$ avec une croissance annuelle de l'ordre de 14 \% (Fig. 1).

Limpact social et relationnel que peuvent avoir une denture équilibrée et un sourire optimal ont participé à l'augmentation de la demande en traitement adulte lors des trente dernières années. Entre 1994 et 2010, les traitements aux États-Unis

\footnotetext{
${ }^{3}$ www.wikinvest.com/stock/Align_Technology_ALGN/Data/ Key_Metrics
} 


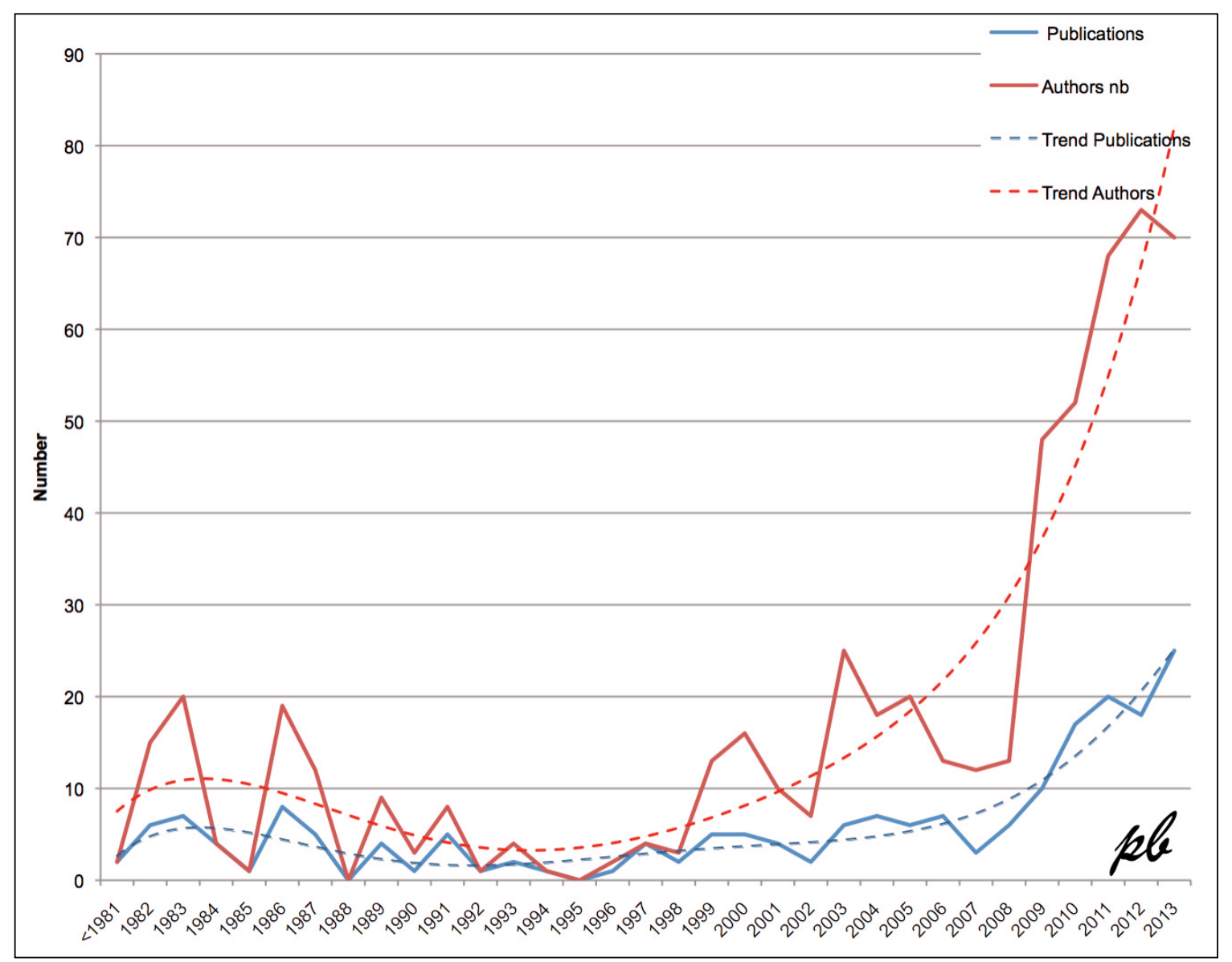

Figure 2

Nombre de publications sur la technique linguale et nombre d'auteurs publiés par année dans les dix principales revues d'orthodontie (Journal of Clinical Orthodontics, American Journal of Orthodontics and Dentofacial Orthopedics, Orthodontie Française, Angle Orthodontist, International Orthodontics, European Journal of Orthodontics, International Journal of Adult Orthodontics \& Orthognathic Surgery, Australian Orthodontic Journal, World Journal of Orthodontics, British Journal of Orthodontics).

sur les patients adultes ont augmenté de $58 \%$ contre seulement $15 \%$ chez les moins de 18 ans [97]. Ceci peut être directement imputable au développement de l'orthodontie vers de nouveaux dispositifs, matériaux, techniques de traitement et de management offrant un appareil compatible avec les soins chez l'adulte, avec des exigences d'efficacité, de confort et de rapidité de traitement. Les brackets vestibulaires en matériau cosmétique ne sont pas perçus par les patients comme véritablement invisibles. Ainsi, en 2014, les principales options d'appareils esthétiques sont l'orthodontie linguale et l'orthodontie par aligneurs transparents. Une revue générale de ces types d'appareils et des caractéristiques des divers produits existant sur le marché nous permet d'aborder les possibilités thérapeutiques de chacun d'eux et leur aptitude à donner une réponse satisfaisante sur le plan esthétique et fonctionnel.

\section{L'orthodontie linguale}

Lorthodontie linguale a développé ces dernières années des concepts thérapeutiques novateurs qui ont modernisé pas à pas l'idée de princeps présentée par Fujita [40] en 1979. Ceci a donné un regain d'intérêt pour une orthodontie esthétique mais trop laborieuse qui avait, au moins un temps, rebuté bon nombre de ses adeptes si l'on en croit la chute du nombre d'auteurs intéressés dans la littérature professionnelle qui atteint son plus bas niveau en 1993. Nous avons exhaustivement comptabilisé par année tous les auteurs et toutes les publications dans les dix principales revues internationales depuis l'existence de l'orthodontie linguale à nos jours (Fig. 2). Il s'avère que les courbes de tendances, aussi bien pour le nombre des auteurs que pour le nombre des articles, suivent les mêmes variations représentant une 


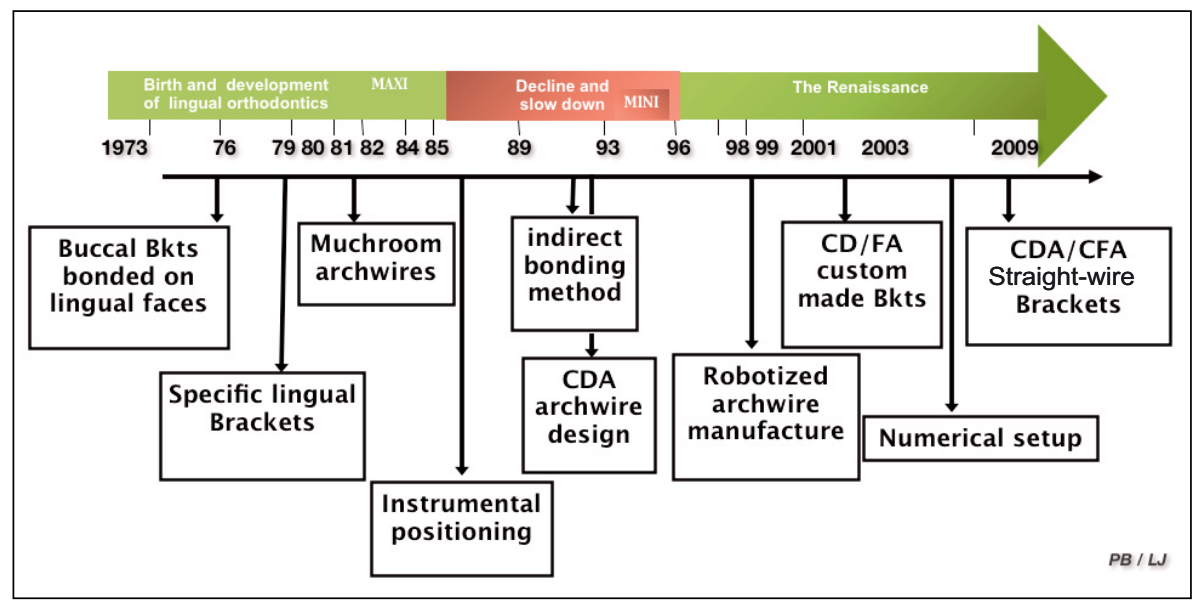

Figure 3

Sauts technologiques en orthodontie linguale (selon brevets et publications jusqu'à 2010).

traduction directe de l'intérêt de la discipline dans la profession. Après un pic en 1982, on observe une décroissance jusqu'en 1993 concomitante avec l'avènement des brackets vestibulaires céramiques qui vont donner l'alternative à une technique décevante quant à ses résultats. Puis, sous l'influence notamment des européens [39, 46, 104], japonais et coréens $[60,61,78,92]$, les procédures de laboratoire se sont ensuite standardisées et les concepts thérapeutiques ont émergé pour un nouveau départ de la technique linguale. Plusieurs méthodes apparaissent et restent encore utilisées à ce jour, notamment pour le placement et le transfert en bouche des attaches. La méthode HIRO [56], du nom de son auteur, est facile à mettre en oeuvre et se réalise à partir d'un set-up sur cire et d'un arc "full size» qui permet le positionnement des brackets et de jauges de transfert individuelles. Le système BEST (Bonding with Equal Specific Thickness) proposé par Fillion en 1989 [40] est une méthode instrumentale qui, à l'aide d'un TARG machine (Torque Angulation Reference Guide : SDS/Ormco, Orange, CA), permet de placer de façon compensée sur le modèle de départ les attaches de telle sorte qu'un arc champignon de taille standard puisse être utilisé lors du traitement. Les diamètres mésio-distaux et les épaisseurs entre la gorge et la face vestibulaire sont retranscrits dans un programme informatique qui imprime la charte d'arc du patient. Le système TOP (Transfert Optimised Positioning), apporté par Wiechmann en 1998, est également instrumental mais procède à partir d'un set-up en cire et les brackets sont placés ici au plus près des faces linguales. Les données répertoriées et les compensations d'épaisseur d'une dent à l'autre sont intégrées cette fois à l'arc qui est fabriqué à l'aide d'un robot.

Ainsi les problèmes initiaux de décollement intempestifs, de précision de collage et d'empâtement de l'appareil ont trouvé des solutions qui ont permis à la discipline de renaître progressivement.

Parmi les dernières avancées significatives, nous devons retenir la présentation de l'arc droit par Takemoto, Scuzzo [119] (2002), l'apparition des brackets customisés par Wiechmann [129] (2003), des propositions d'application en imagerie proposées par Fillion [38] en 2005. À partir de là, une forte croissance s'opère après 2007 soutenue par le rachat et la commercialisation par 3M-Unitek du système Incognito, ainsi que par plusieurs autres propositions thérapeutiques alternatives.

On constate que l'amélioration s'est faite conjointement dans deux directions : sur le matériel d'une part, sur les techniques de transfert d'autre part.

Le déterminant de cette évolution a été l'informatisation croissante des procédures avec une $\mathrm{CAO}$ (Fig. 3) qui commence en 1989 par le dessin des arcs (logiciel DALI pour Dessin d'Arc Lingual Informatisé), passe par la fabrication des arcs en 1998, la conception des brackets en 2003 [93], le set-up numérique en 2005 [37], intéresse l'ensemble de la chaîne en 2009 (système LJ) [10].

Avec Pernier, et al. [101], on peut alors distinguer trois types d'évolution:

- la sophistication des attaches de l'industrie, qui a fait migrer le bracket Génération7 de Kurz 
longtemps leader jusqu'à des brackets plus réduits, plus profilés et auto-ligaturants,

- la divulgation d'une orthodontie linguale simplifiée et peu coûteuse réservée aux traitements minimes,

- les systèmes dits « customisés » avec des attaches complètement individualisées pour chaque dent de chaque patient.

Nous ne saurions donner ici le catalogue exhaustif de tous les systèmes d'orthodontie linguale existant ou ayant existé. Seules les conceptions abouties et commercialement viables sont appelées à subsister. Ces systèmes n'ont pas tous les mêmes objectifs, occupent chacun une niche qui correspond à une utilisation plus ou moins étendue et sont construits à partir d'attaches spécifiques.

\subsection{Les brackets linguaux préfabriqués}

Parmi les nombreuses attaches ayant vu le jour, nous ne décrirons ici que les représentantes des principales espèces. Lévolution s'est faite au cours du temps sur le volume de l'attache, sur sa base, sur l'adaptation à plusieurs groupes de dents. Elles nécessitent un transfert en bouche par collage indirect avec une étape de laboratoire utilisant une méthode de montage instrumentale ou numérique.

- Le bracket initial de Kurz [113, 114] : plusieurs versions de ce bracket se sont succédées, respectant ses caractéristiques principales héritées de sa conception de princeps : une base adaptée au relief lingual des dents, une gorge incorporant un torque correspondant, un plan de morsure. La gorge est horizontale pré-informée, disponible en $.018 \times .025$ ou en $.022 \times .028$. Cette attache a fait pendant longtemps référence et sa diffusion universelle a été servie par les promoteurs de l'orthodontie linguale à ses débuts que furent Jack Gorman et autres membres de la «task force » d'Ormco.

- Le bracket de Hiro [55] : en 1999, Hiro propose un nouveau prototype dont le but est de résoudre les principaux problèmes posés par les brackets initiaux. Ce bracket est plus réduit, plus arrondi, d'épaisseur moindre, sans plan de morsure, comporte une gorge à $45^{\circ}$ de torque, un treillis moins serré avec une surface de la base diminuée (Fig. 4). Le concepteur obtient d'excellents résultats avec ce matériel et constate une réduction de l'inconfort des patients sur l'élocution, les irritations linguales, les

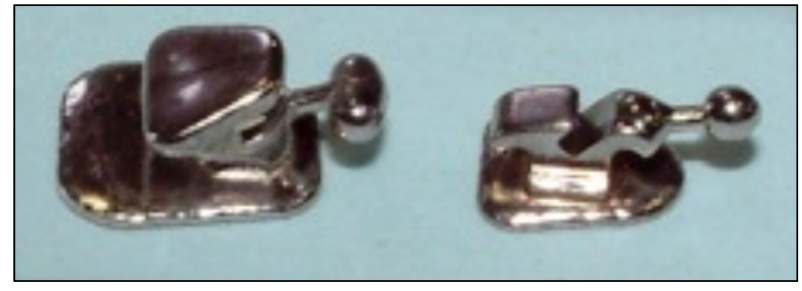

Figure 4

Comparaison du bracket de Kurz et du bracket de Hiro.

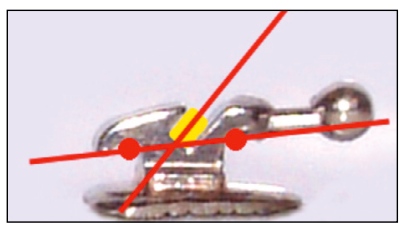

a

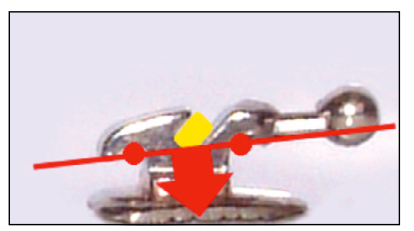

$\mathrm{b}$

Figure 5

(a et b) Vue latérale du bracket de Hiro. La gorge est à $45^{\circ}$ permettant un bon maintien de l'arc par la ligature (avec la courtoisie du Dr Toshiaki Hiro).

interférences occlusales. Par ailleurs, il n'est plus nécessaire de ligaturer par une «double over-tie » et la fréquence de décollement diminue (Fig. 5).

- Le magic bracket [117] : il diffère des précédents par sa composition en Cr-Co en une seule pièce, sans soudure de sa base dont l'intrados est sculpté au laser. Il en résulte une excellente biocompatibilité théorique et une bonne rétentivité. Ses contours sont arrondis, le plan de morsure étendu et les crochets en continuité avec le corps du bracket. Il existe plusieurs modèles par type de dents et par côté pour les canines, déclinés en différentes largeurs. Plusieurs options concernent les tubes molaires dont certains sont convertibles. Mais son originalité vient de plusieurs de ses caractéristiques : une gorge edgewise en $.020 \times .020$, horizontale à insertion «en porte-manteau » dans la région antérieure, verticale dans la région prémolo-molaire. Cette disposition conçue par Sorel a été dictée par des exigences bio-mécaniques permettant une optimisation du contrôle des informations de l'arc dans les trois ordres sur toutes les zones de l'arcade. En revanche, le principe d'insertion requiert une taille 


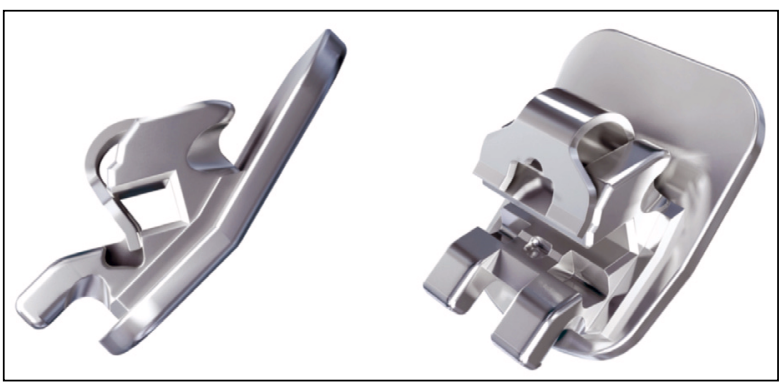

Figure 6

Vue latérale et de trois-quart du bracket In-Ovation L avec clip en position fermée et ouverte.

relativement importante des brackets et une ligature «double over-tie ».

- Le STb [109] : développée par Scuzzo et Takemoto en 2003, cette attache est censée garantir plus de confort à la fois pour le patient et pour le praticien. Il s'agit d'un bracket miniaturisé, de 1,5 mm d'épaisseur, dont la réduction a des répercussions directes sur l'encombrement en bouche, sur la distance inter-bracket qui se trouve augmentée, sur le contrôle de la mécanique au fauteuil rendu plus difficile en raison de sa faible taille. La gorge est en $.018 \times .025$, le bracket est étroit, mono-plot, décalé gingivalement sur sa base, avec des ailettes antirotations sur les prémolaires. De par sa conception, cette attache convient parfaitement à une mécanique basse-friction, avec des forces légères et son positionnement gingival peut permettre un montage compatible à l'usage d'un arc droit.

- Le bracket autoligaturant In-Ovation L [95] (Fig. 6) : deux raisons principales viennent à l'esprit dès que l'on évoque un système auto-ligaturant. La réduction des forces de friction et la facilité d'utilisation sont les deux arguments majeurs qui sont généralement mis en avant pour ce type de dispositifs. La technique linguale n'échappe pas à la règle. La réduction de la distance inter-bracket rend l'insertion et le maintien de l'arc difficile dans les cas d'encombrement par exemple et un boitier auto-ligaturant permet de gagner un temps appréciable lors de la solidarisation de l'arc. Sur le même principe que ces attaches auto-ligaturantes vestibulaires, la société Densply GAC international (USA) a créé un bracket lingual de petite taille, 1,5 mm d'épaisseur pour 2,2 $\mathrm{mm}$ de large, bi-plot, avec une gorge horizontale en $.018 \times .025$. La base est dessinée pour s'adapter à la partie la plus déclive des fosses linguales des dents antérieures et peut être courbée à la demande, au laboratoire, pour un positionnement optimal avec un minimum d'épaisseur de collage. Le clip, à double mécanisme, vertical puis à rotation, rend son épaisseur compatible à un usage lingual et fait office de plan de morsure. Cependant, il faut noter qu'il s'agit d'un bracket semi-actif, le clip n'étant en action sur l'arc que pour des tailles élevées et à faible degré de friction pour des tailles inférieures.

\subsection{Les techniques simplifiées}

Afin de mettre en place des solutions proportionnées aux traitements minimes, de nombreux auteurs ont proposé des techniques linguales simplifiées. Dépourvues de contrôle tridimensionnel, ces techniques permettent au plus d'aligner les dents en mettant en oeuvre des procédures directes pratiquement sans phase de laboratoire. Nous avons retenu ici les plus pratiquées.

- La méthode 2D [21, 22] : développées par Philippe en 1984, les attaches 2D ont bénéficié d'une extension de gamme en 2005 avec plusieurs modèles de brackets qui restent fidèles au concept de base. Le medium Twin est universel, proche du bracket initial, le large Twin pour corriger les rotations, le narrow Single pour les cas avec fort encombrement, le 2D Plus avec un point d'appui supplémentaire pour délivrer un torque actif. Ces divers types peuvent comporter un crochet gingival ou en T. La caractéristique de ces attaches est de ne pas comporter de gorge. Larc s'insère verticalement dans des ergots en position ouverte qui sont rabattus à la pince de Weingart pour le fixer. Louverture se fait en sens inverse avec une spatule et peut s'avérer difficile et génératrice de décollement. L'absence de gorge permet d'avoir une épaisseur de seulement 1,4 mm. La liaison arc-bracket reste peu précise et ne permet pas un contrôle direct du torque. Le collage est effectué en méthode directe, en unitaire ou avec gouttière, sans intégrer les différences d'épaisseur des diamètres bucco-linguaux. Cependant, la technique $2 D^{\circledR}$ (Forestadent, Pforzheim, Germany) permet une approche peu coûteuse, sans recours à des procédures de laboratoire sophistiquées, avec des principes mécaniques simples, des forces légères délivrées par des arcs ne pouvant excéder $.022 \times .016$. En revanche, le résultat dépend directement de l'habileté du praticien au fauteuil à coller et à plier l'arc en fonction du déplacement souhaité. 
Les indications restent donc limitées à des traitements généralement de courte durée :

- récidive discrète de traitement,

- fermeture d'espaces et diastèmes mineurs,

- correction de petits encombrements,

- correction d'articulé inversé unitaire,

- correction de dystrophies modérées,

- correction de supraclusion.

Cette technique représente une alternative à des cas de technique conventionnelle et peut être réservée à des adultes ne présentant pas de malocclusion sévère, dont la correction ne nécessite pas un contrôle rigoureux notamment du troisième ordre.

- Le STb «social-six» [79] : il s'agit d'un concept thérapeutique développé par Scuzzo et Takemoto [108] destiné aux traitements des encombrements faibles à modérés et aux fermetures d'espaces dans la région antérieure. Le traitement est réputé être effectué en un temps court de l'ordre de 6 à 16 semaines, en utilisant une ou deux paires d'arcs. Les brackets employés sont des STb sur les six dents antérieures pouvant s'étendre dans certains cas jusqu'à la première prémolaire. Le collage est effectué à l'aide d'une gouttière de transfert réalisée sur le modèle de la malocclusion. Selon les promoteurs, l'utilisation de forces légères produites par un arc sous dimensionné $012 \mathrm{NiTi}$ assure la rapidité des mouvements. Les finitions peuvent être alors effectuées par des arcs plus rigides en 016 NiTi ou TMA.

\subsection{Les systèmes individualisés par CAO/CFAO}

Lorthodontie est rentrée dans une nouvelle ère où la technologie numérique joue un rôle croissant en direction de la personnalisation complète des dispositifs thérapeutiques. La technique linguale a franchi le cap loin devant avec le premier système de fabrication de bracket en CFAO introduit par Wiechmann en 2003 [128, 129]. D'autres systèmes similaires ont vu le jour depuis avec des variantes concernant le mode de fabrication, le matériau, le type d'informations en entrée et en sortie du processus de fabrication. Incognito ${ }^{\circledR}$ (Top Service $3 \mathrm{M}$ Unitek) reste le représentant hégémonique de ce type d'appareil; certains apparaissent, comme Harmony ${ }^{\circledR}$ (distribué par American Orthodontics), d'autres disparaissent comme Lingualjet ${ }^{\circledR}$, d'autres naissent comme Win Apparatus (DW Lingual System, Bad Essen, Germany) ou commencent à s'établir comme e-braces ${ }^{\circledR}$ (Guangzhou Riton Biomaterial, China) ou Lingualmatrix ${ }^{\circledR}$ (Mumbai, India).

Ils présentent plusieurs points communs:

- l'utilisation d'un modèle virtuel sur lequel s'opère la conception des brackets en relation avec l'anatomie de chaque dent,

- une base étendue sur une large surface de collage englobant intimement l'anatomie linguale de la couronne correspondante (Fig. 7),

- la construction numérique du corps du bracket selon un procédé spécifique ou son importation à partir d'une bibliothèque numérique,

- une procédure identique pour les accessoires, ailettes, crochets, marquage du bracket,

- l'exportation des brackets virtuels vers un périphérique de conversion: machine outil (fraisage multi-axe par un système CNC (computerized numerical control)), imprimante 3D (Rapid Prototyping machine) produisant des maquettes calcinables à couler, apposition ou frittage laser (Selective Laser Sintering) qui représente aujourd'hui la technologie la plus avancée.

Ils diffèrent sur plusieurs caractéristiques :

- le type de matériaux : alliage d'or, acier ChromeCobalt, acier Nickel-Chrome,

- le set-up de travail : sur modèle (cire et plâtre), ou set-up virtuel effectué avec une aide logicielle plus ou moins sophistiquée,

- le type d'arc : arc champignon, arc robotisé, arc droit,

- le type de bracket : insertion horizontale ou verticale, auto-ligaturant ou non pour la plupart,

- l'intrados de la base : traitements physicochimiques, bases «mesh», accessoires de rétention.

Le succès majeur de ces techniques tient essentiellement à trois raisons :

- Lamélioration du collage : l'absence d'épaisseur compensatoire en composite, la base métallique étendue dessinée sur mesure au relief de la dent, donnent à l'attache une grande stabilité et une résistance accrue au décollement [8] ; allié à la gouttière de transfert, ceci permet un collage indirect de grande précision; également en cas de décollement ou de collage unitaire différé, le praticien peut utiliser une méthode directe avec une précision acceptable puisque l'attache va venir se 


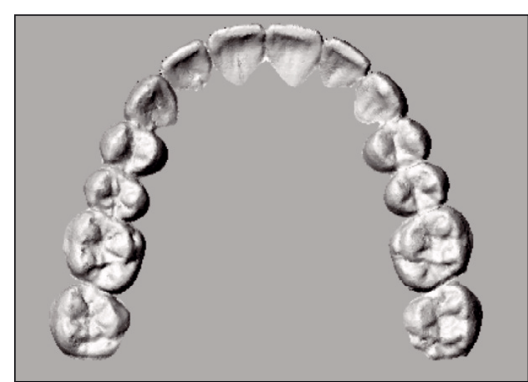

a

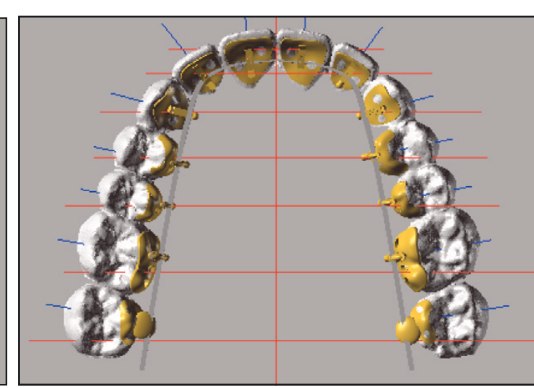

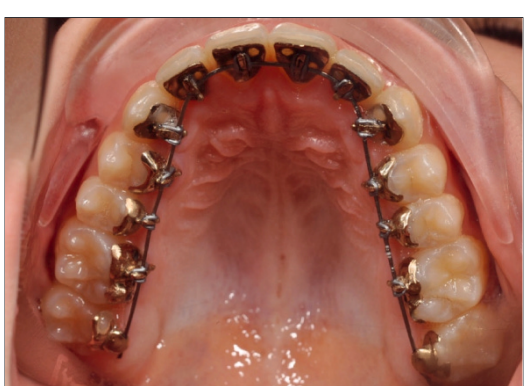

C

Figure 7

(a) set-up numérique, (b) appareil LJ virtuel désigné avec bases étendues, (c) appareil LJ en bouche après la phase d'alignementnivellement.

plaquer sélectivement sur la dent dans sa position adéquate.

- La mécanique de l'arc : l'information est dans l'arc (arc robotisé), dans le bracket (arc droit) ou dans les deux (techniques mixtes). Le principe de fabrication d'attaches individualisées garantit une relation arc/gorge optimale permettant d'incorporer des informations d'hypercorrection en fonction des impératifs thérapeutiques. Ceci a un retentissement sur toutes les phases du traitement et permet en particulier de diminuer les problèmes de finition.

- La miniaturisation des attaches et le décalage du corps du bracket sur sa base permettent de limiter les interférences et l'inconfort pour le patient en absorbant dans la masse du bracket les variations anatomiques des arcades et de la surface linguale des dents rencontrées.

$\mathrm{Au}$ total, cette orthodontie linguale numérique constitue un saut technologique aujourd'hui irréversible qui donne à la technique une nouvelle marge de progression avec des perspectives de généralisation de la discipline. On est passé, à beaucoup de similitudes près, de l'art à l'industrie.

\subsection{Discussion}

Lorthodontie linguale se décline donc en toute une variété de dispositifs qui vont répondre à des exigences cliniques, techniques, économiques différentes selon les patients et leur praticien. Devant des propositions relativement différentes et plus ou moins sophistiquées, il convient d'éclairer le choix à effectuer. Les conséquences et les résultats de l'orthodontie linguale ont été étudiés selon plusieurs aspects et à divers moments de sa courte histoire. Deux éléments se dégagent comme facteurs d'appréciation selon que l'on se place respectivement du côté du patient ou du côté du praticien.

\subsubsection{La perception des patients}

Quel que soit le modèle de bracket utilisé, la littérature rapporte toujours le même type de problèmes liés à l'inconfort du dispositif :

- des irritations linguales,

- des troubles phonatoires,

- des difficultés d'hygiène,

- des modifications masticatoires avec des gênes alimentaires,

- des douleurs.

C'est le prix à payer pour une orthodontie invisible! Une étude comparative entre technique linguale et vestibulaire [24], sur deux groupes d'adolescents n'a pas montré de différence observable concernant l'hygiène, la gêne alimentaire ou la douleur. En revanche, les problèmes phonatoires et le temps d'adaptation apparaissent en défaveur de l'appareil lingual. Ce type de difficultés, minimisé par des études antérieures $[3,39,41,112]$, est confirmé par des études récentes à méthodologie plus rigoureuse, ainsi que dans une méta-analyse, comme étant le point le plus négatif des dispositifs linguaux [80]. Les phonèmes en /s et en /t sont les plus perturbés. Une étude prédictive sur l'altération de la prononciation [127] suggère que l'espace disponible pour la langue est plus affecté dans les cas de faible valeur de $\mathrm{SNB}$. De plus, la taille et le positionnement des brackets joueraient un rôle sur la perturbation de l'élocution $[57,58]$. Les irritations et douleurs des tissus mous, et de la langue en particulier, sont également constatées par divers travaux avec une différence statistiquement significative $[22,31,66,88]$ vis-à-vis de la technique vestibulaire. Elles peuvent se prolonger 
jusqu'à trois mois. La gène masticatoire apparaît de façon moins nette selon les études; elle est de règle et sévère à la pose de l'appareil lingual pour s'atténuer dans les premiers mois du traitement. Des rétentions alimentaires plus nombreuses qu'en vestibulaire ont été mises en évidence $[24,25,113]$.

Le seul facteur positif pour le patient reste l'invisibilité de l'appareil qui apparaît comme une condition sine qua none pour plus de la moitié des adultes en traitement $[13,41,99]$. Parmi eux, la femme de moins de 40 ans se dégage dans la littérature [16,40, 58] comme correspondant à la typologie préférentielle du patient en orthodontie linguale.

Ainsi, la plupart des auteurs [40, 101, 108, 112] préconisent d'informer objectivement les patients sur les désagréments de la technique linguale concernant notamment l'élocution et la gêne encourue notamment en début de traitement.

\subsubsection{La perception des praticiens}

Le diagnostic des échecs de l'orthodontie linguale à son lancement a pointé la nécessité d'améliorer le collage des attaches linguales qualitativement en précision et quantitativement en résistance au décollement. Les améliorations matérielles et techniques qui se sont succédées ont donné à l'orthodontiste aujourd'hui un appareil globalement fiable qu'il s'agisse de brackets du commerce pré-fabriqués ou de systèmes CFAO sur mesure. Cependant, des écueils subsistent dans le maniement de l'appareil :

- le temps au fauteuil est augmenté,

- la mécanique est moins favorable,

- les finitions sont plus difficiles.

L'ergonomie du dispositif est essentielle pour limiter le temps de travail qui s'opère pour le praticien dans une position inconfortable et vite fatigante. La commodité d'insertion de l'arc, la facilité de le ligaturer au bracket, la mise en place aisée de chaînettes élastomériques, la résistance aux décollements provoqués par toutes les manœuvres précédentes sont autant d'éléments déterminants pour le confort du praticien. Respectivement, l'utilisation d'un arc droit, de brackets auto-ligaturants, l'existence de crochets et bi-plots, de bases « customisées » sont des réponses adaptées. Elles affectent, en revanche, fortement le confort du patient.

Le contrôle de la thérapeutique est un autre point crucial. Il dépend de la compréhension du système mécanique, mais est intimement lié au type d'appareil qu'utilise le praticien. Comme il existe une typologie préférentielle de patient, certains experts Gorman [50], Scuzzo ou Takemoto [108, 112] ont décrit une malocclusion typique pour débuter en orthodontie linguale. Il s'agirait d'une classe II division 2 modérée avec indication d'extraction des premières prémolaires supérieures. Le contrôle de la mécanique est facilité par les contacts inter-incisifs de même que la correction de la supraclusion. Les avis divergent sur la question et certains comme Hiro [55] recommandent plutôt, pour un premier cas, de commencer par une classe II division 1 dont les exigences mécaniques seront moindres. Tout dépend en fait du positionnement des attaches sur la face linguale et si le profil des brackets comporte un plan de morsure. En fonction de l'avancée du traitement, plusieurs phases cliniques demandent une approche différenciée en technique linguale :

- Lalignement peut se faire avec une certaine difficulté compte tenu, d'une part, de la proximité des attaches qui, en cas d'encombrement, est encore plus critique [45]. Il devient alors difficile de passer le premier arc, a fortiori s'il s'agit d'un arc champignon. Il est également délicat dans ces conditions de fermer les clips d'un bracket autoligaturant. L'utilisation d'arcs ronds de faible section, de matériaux thermiques et à limite élastique élevée, type $\mathrm{Cu}-\mathrm{Ni}$-Ti, seront des recours possibles. Des artifices comme les ligatures en lasso, doubles "over-tie », ligatures tire-pousse, «by-pass » séquentiel pourront aussi servir. La solution de l'insertion verticale de l'arc (incognito, magic) est tentante mais se fait au détriment du contrôle du deuxième ordre qui peut alors s'avérer préjudiciable dans la zone antérieure de l'arcade. L'insertion à $45^{\circ}$ du bracket de Hiro constitue selon son inventeur une solution à mi-chemin. D'autre part, à l'inverse d'un arc vestibulaire qui pourra coulisser librement au fur et à mesure du redressement et de la correction des rotations, l'arc lingual se retrouve contraint et ne peut sans activation spécifique opérer d'action d'expansion. Les techniques favorisant des déplacements basse-friction (LSWA : Lingual Straight Wire Appliance) sont théoriquement plus efficientes $[9,37,109,119,120]$.

- Concernant le contrôle du torque, il doit s'exercer dès le nivellement, notamment dans les cas 
d'incisives en forte palato-version initiale. De par les conditions bio-mécaniques résultant de la technique linguale $[47,110]$, l'emploi d'arc de forte section ou l'utilisation d'accessoires est nécessaire pour ne pas induire de torque négatif pendant cette phase.

- En thérapeutique linguale, le contrôle du deuxième ordre est intimement lié aux effets de torque dans la région antérieure [47]. Ceci est bien visible dans les cas de rétraction incisivocanine avec ou sans extraction de prémolaires. Il peut apparaître à l'activation d'une mécanique de recul incisivo-canin un fléchissement de l'arc dans le sens vertical connu sous le nom d'effet «bowing », qui s'exprime ici verticalement. Il va se produire une égression de l'incisive, une mésioversion molaire avec un torque actif négatif dans la zone antérieure. La taille et la rigidité de l'arc, la clairance de l'arc dans la gorge, ainsi que le mode d'activation avec des courbures de compensation interviennent dans le contrôle du déplacement. Les mini-vis d'ancrage palatines sont aussi des accessoires précieux pour limiter les effets parasites en faveur d'une mécanique préservant l'ancrage postérieur [9].

- Cet effet «bowing» peut se manifester aussi dans le sens transversal et fera appel aux mêmes solutions. Lusage d'un arc droit va permettre de bien limiter ce phénomène en évitant des plicatures, en réduisant la distance d'arc inter-bracket, en évitant des translocations dans le réseau cristallin du matériau et en favorisant le glissement [7]. Les arcs robotisés, les arcs champignons, avec des in-set prémolaires, ne remplissent pas ces conditions. Compte tenu de leur anatomie radiculaire, les molaires maxillaires auront une propension à la rotation mésio-vestibulaire dès que l'on sollicite leur ancrage. Des thérapeutiques avec minivis permettront ici aussi de s'affranchir du problème $[7,75]$.

La précision des finitions est directement liée à la fois à la qualité et à la stabilité du résultat. De plus, pour le traitement des adultes, l'environnement parodontal, endodontique, la présence d'abrasion, de reconstructions prothétiques, de sites d'extraction anciens, ainsi que toute l'histoire dentaire du patient va augmenter d'autant les difficultés de finitions [49]. Deux éléments particuliers concernent les appareils linguaux : le positionnement des attaches et la spécificité de la thérapeutique.

Qu'ils soient collés avec des méthodes de laboratoire ou qu'ils fassent appel à des systèmes de CAO/CFAO, les appareils linguaux, techniques simplifiées mises à part, sont aujourd'hui transférables en bouche avec une précision acceptable. Cette précision est nécessaire à la validité de la technique employée dont la cohérence dépend du bracket, du type d'arc, du positionnement du bracket sur la dent. Dans ce contexte, le recollage en cas de perte accidentelle doit être aussi rare que précis. Le positionnement gingival des attaches permet l'élaboration d'une technique d'arc droit mais aura en contrepartie une distance inter-bracket plus réduite. La miniaturisation des brackets ou les bases métalliques « customisées » offrent cette possibilité. La distance de la gorge au centre de résistance est diminuée pour les dents antérieures qui sont l'objet de toutes les attentions. A contrario, une position plus centrée sur la couronne à mi-hauteur et au plus près de la surface linguale augmentera le périmètre de l'arc dans cette zone, mais nécessitera un arc informé comportant des courbures de compensation seulement réalisables en pratique par robot (Orthomate ${ }^{\circledR}$ system, Orametrix, Dallas, TX).

Les arcs de finitions, réalisés par l'orthodontiste, demandent évidemment au praticien un temps au fauteuil supplémentaire. Mais celui-ci pourra incorporer à sa guise les informations « à la pince » à partir d'arcs droits ou d'arcs champignons préformés. Ceci donne beaucoup plus de flexibilité et de réactivité pour parfaire le résultat tout en restant maître de la conception. Ces finitions peuvent être parfaitement soignées et préparées à l'avance en réalisant une empreinte des arcades une fois que les objectifs généraux du traitement sont atteints [48]. Il va de soi que les phases cliniques précédant l'étape de finition doivent être parfaitement contrôlées, notamment dès le début de traitement, afin d'éviter les effets indésirables de la mécanique linguale et de minimiser ainsi l'étape d'intercuspidation/finitions.

\subsection{Cas cliniques}

\subsection{1. $\operatorname{Cas~}^{\circ} 1$}

Coralie présente une classe III squelettique et dentaire, une légère asymétrie mandibulaire, des compensations alvéolaires, un encombrement aux 


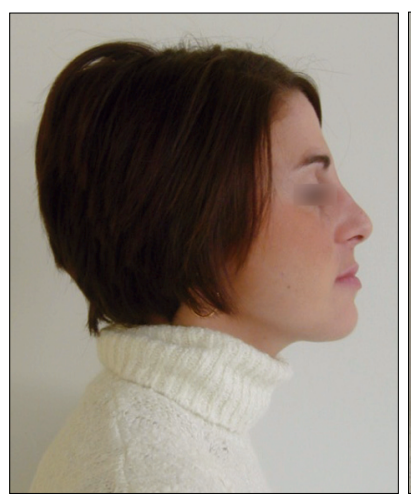

a

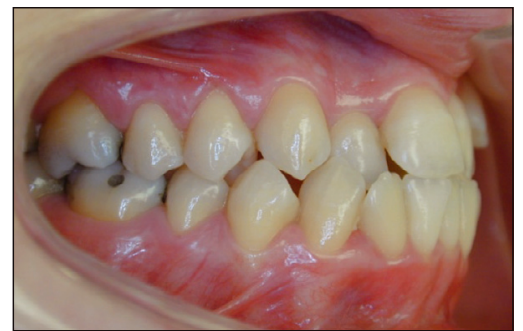

d

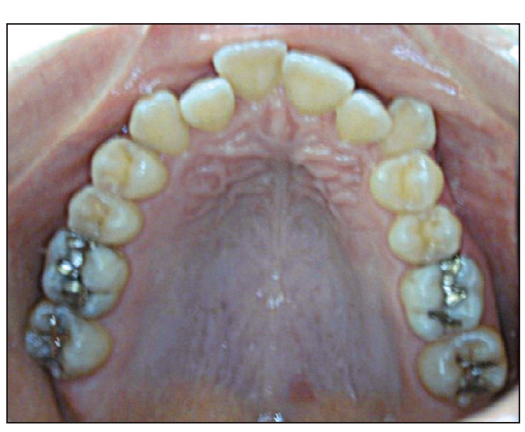

g

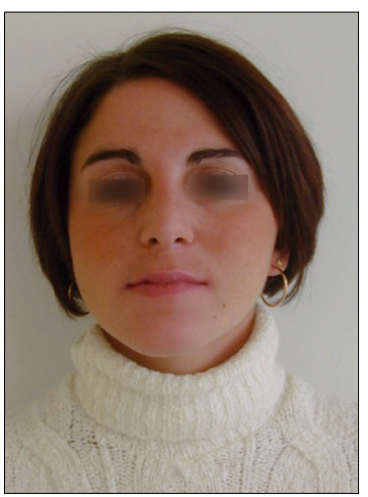

b

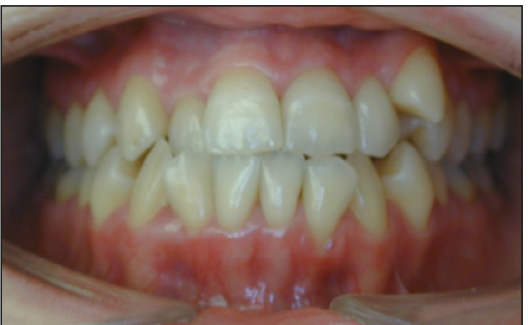

e

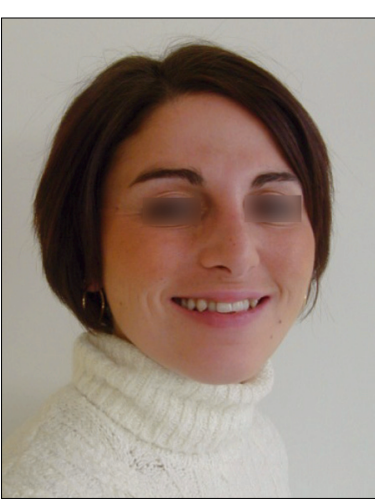

C

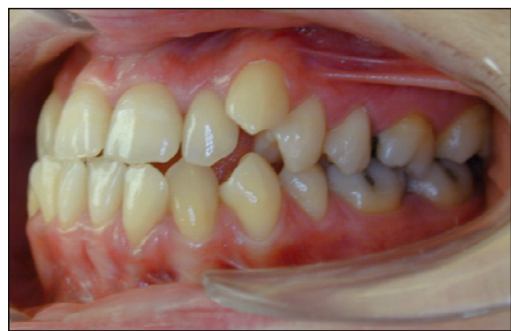

f

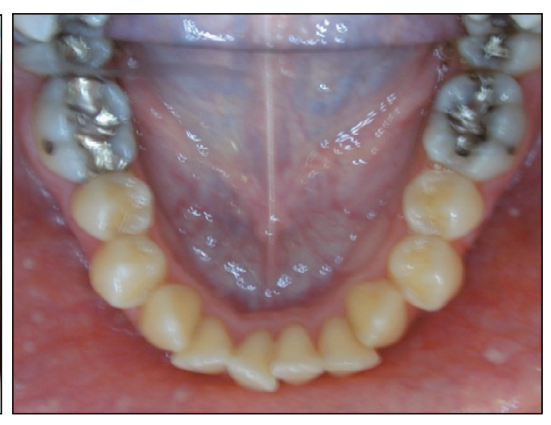

$\mathrm{h}$

Figure 8

Vues exo- (a à c) et endo-buccales ( $\mathrm{d}$ à h) avant traitement.

deux arcades, une déviation des médianes et un articulé antérieur en bout à bout. Les répercussions faciales vont bien au delà de l'encombrement incisif et de la dystopie de la 23 qui l'amènent à consulter en 2001 (Fig. 8). Elle travaille dans la communication et souhaite une solution esthétique. Un traitement en technique linguale lui est proposé en vue de la résolution chirurgico-orthodontique de sa malocclusion.

Les objectifs de traitement sont la restauration de l'équilibre facial, la normalisation de l'occlusion, une stabilité du résultat à long terme. La préparation prévoit l'extraction des $14,24,35,45$, la fermeture des espaces, la stabilisation et la coordination. Une imagerie 3D pré-chirurgicale est planifiée avant la fin de la préparation. La chirurgie est programmée avec une double ostéotomie, Lefort 1 d'avancée et Epker de dé-rotation mandibulaire (Figs. 9 et 10).

Les brackets utilisés sont des brackets de Kurz (Génération 7, Ormco) en .018 × .025. Le système de positionnement est réalisé par TOP Service.

Le collage est effectué en méthode indirecte par une gouttière de transfert en silicone lourd semi-rigide. La colle utilisée est un composite liquide double mélange, auto-polymérisable (MaxiCure, Reliance $^{\mathrm{TM}}$, Itasca, IL). Le collage des deux arcades est fait dans le même temps, les extractions sont effectuées après collage (Fig. 11). Les interférences entre les deux groupes incisivo-canins provoquent une infraclusion antérieure. Le premier jeu d'arc est une paire d'arc thermique en 


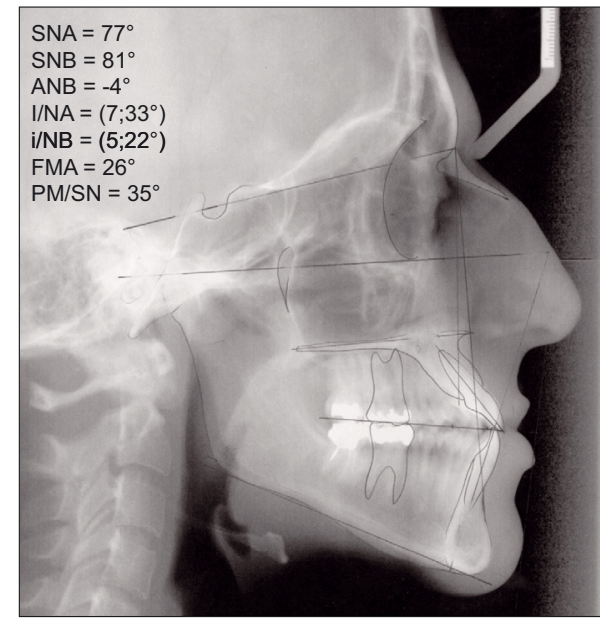

a

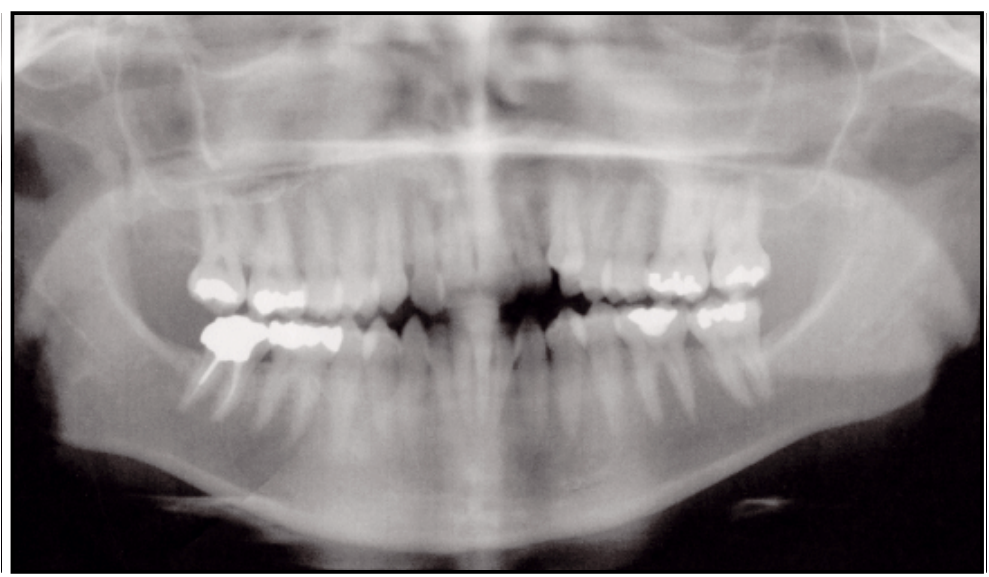

b

Figure 9

Radiographies avant traitement.

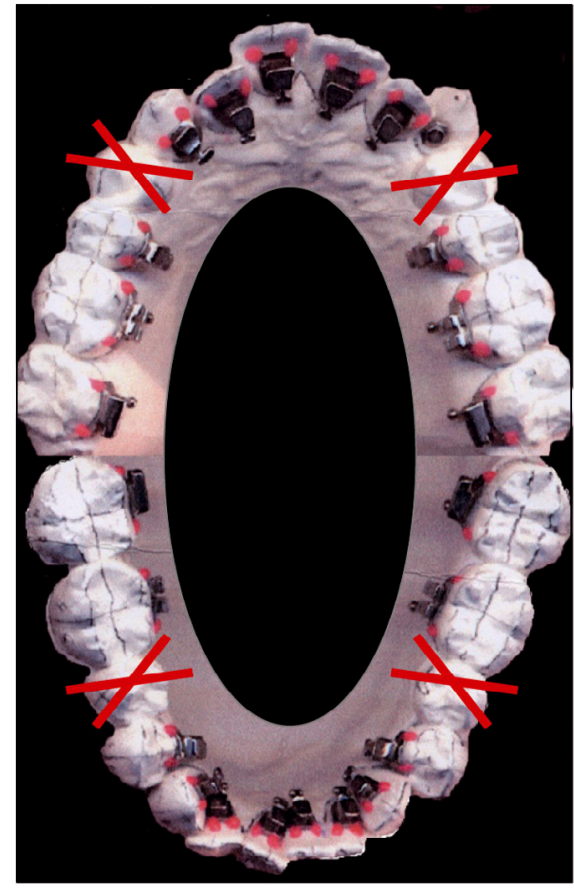

Figure 10

Positionnement des brackets (T.O.P. Service).

Cu-Ni-Ti .016 × .022, confectionné par robot, qui sont ligaturés en ligatures simples puis en double over-tie secondairement. Les arcs commandés suivants sont en $\mathrm{Cu}-\mathrm{Ni}-\mathrm{Ti} .017 \times .025$, la phase d'alignement/nivellement est poursuivie et le recul des 13 et 23 amorcé (Fig. 12). À ce stade, le collage différé de la 23 peut être effectué (Fig. 13).

Après 15 mois de préparation, une acquisition TDM est demandée afin d'effectuer une analyse de
Treil pour parfaire la préparation. La reconstruction 3D des structures dentaires et de la charpente maxillo-faciale permet d'affiner la planification de la chirurgie et la fin de la phase orthodontique (Fig. 14 et 15).

En fin de préparation, des brackets céramiques faisant office de plots pré-chirurgicaux sont collés sur les secteurs latéraux des deux arcades. Ils vont servir uniquement au blocage per opératoire et à la pose des élastiques intermaxillaires post-chirurgicaux (Fig. 16, 17). Les brackets en céramique préencollés présentent l'avantage d'être pratique d'utilisation, d'être esthétiques et de bien résister au décollement pendant les manoeuvres chirurgicales en cours d'intervention.

Après réduction de l'oedème post-chirurgical, les finitions vont pouvoir être entreprises à partir des arcs en place. Il s'agit d'arcs confectionnés par robot sur lesquels on va appliquer à la pince, avec beaucoup de précautions pour éviter leur fracture, les compensations supplémentaires nécessaires (Fig. 18). Des élastiques verticaux sont portés du côté lingual, d'abord sur les arcs complets qui seront sectionnés pour finir avec des élastiques et des arcs sectionnels antérieurs (Fig. 18). L'occlusion terminale est conforme à nos objectifs. Esthétiquement, juste après la chirurgie, les rapports faciaux sont harmonieux (Fig. 19). Les documents quatre ans après montrent une bonne stabilité du résultat à long terme (Fig. 20 et 21). Seule une contention collée sur 33 et 43 a été maintenue. 


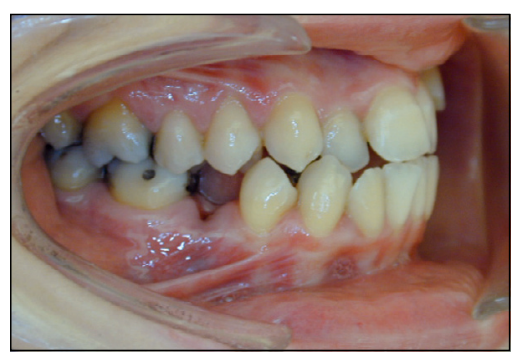

a

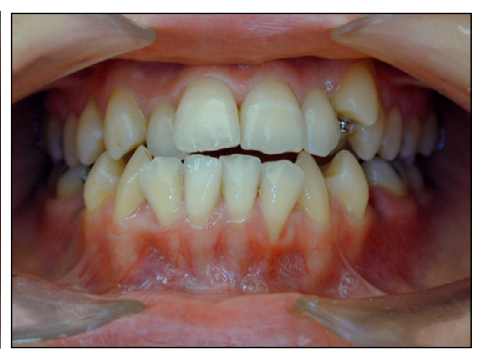

b

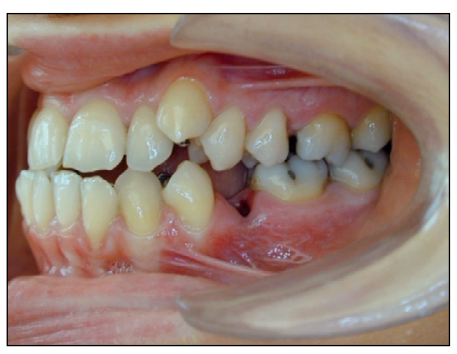

C

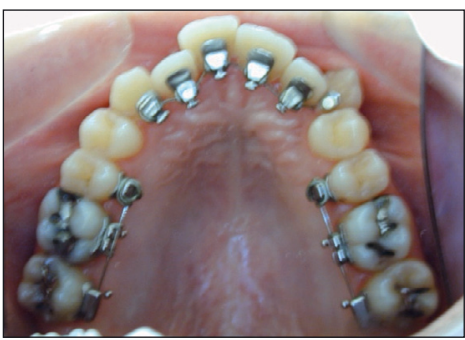

d

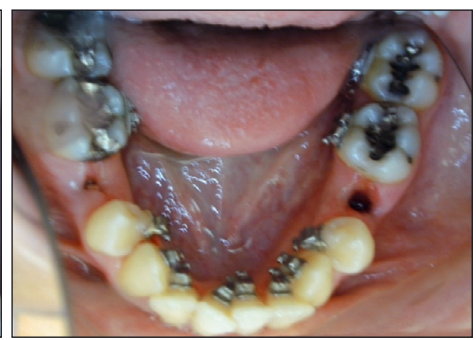

e

Figure 11

Rapports occlusaux après collage des deux arcades.

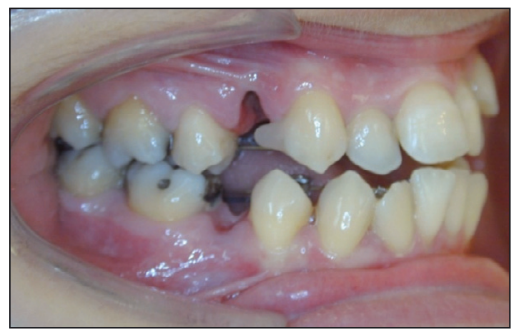

a

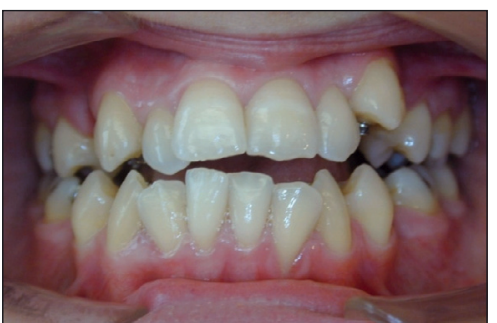

b

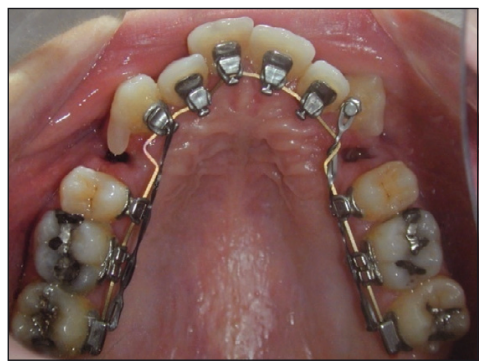

d

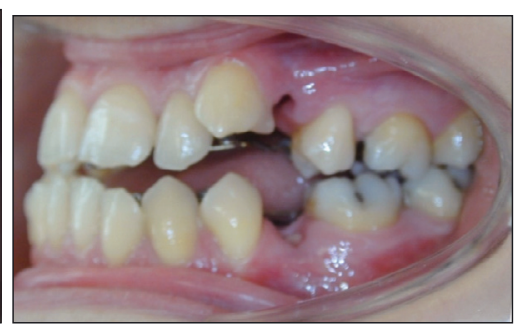

C

Figure 12

Après 3 mois de traitement - début du recul canin.

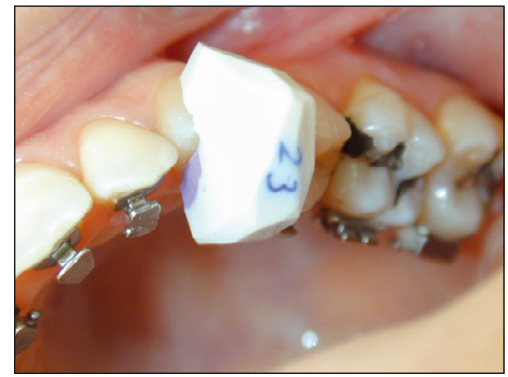

Figure 13

Collage différé de la 23 par transfert individuel. 


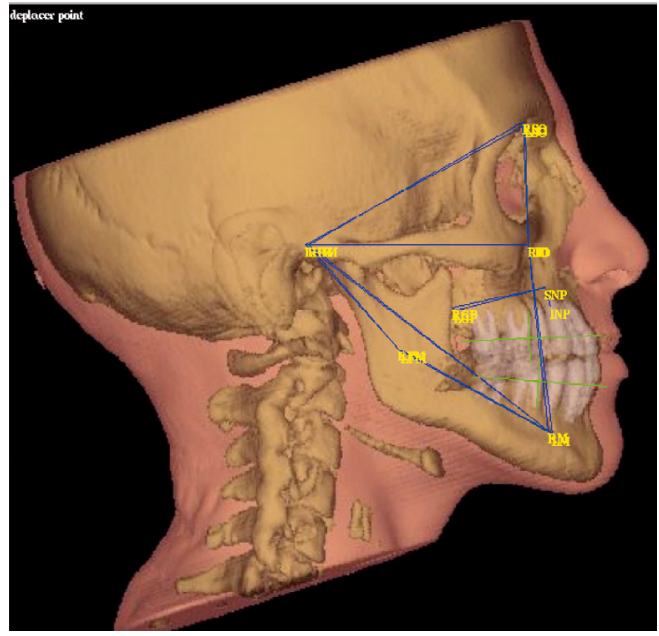

a

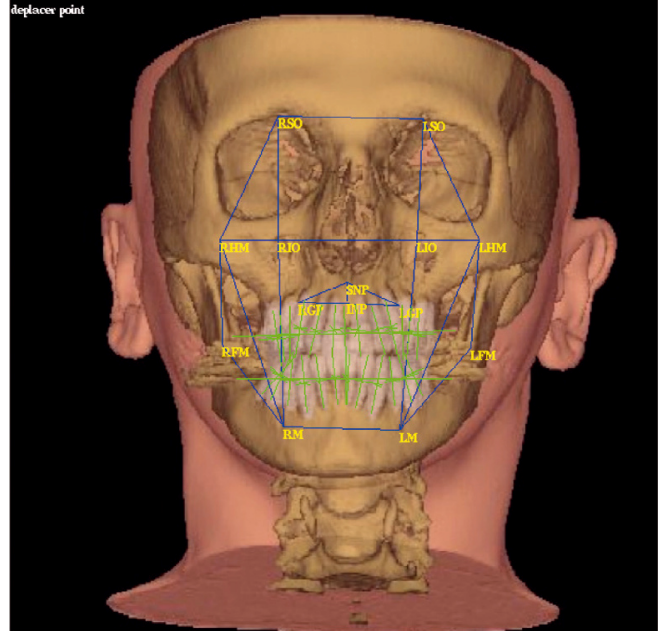

b

Figure 14

Analyse Cepha3DT en vue latérale droite (a) et de face (b) : la charpente maxillo-faciale objective les rapports basaux de classe III et l'asymétrie mandibulaire verticale par léger excès antérieur gauche.

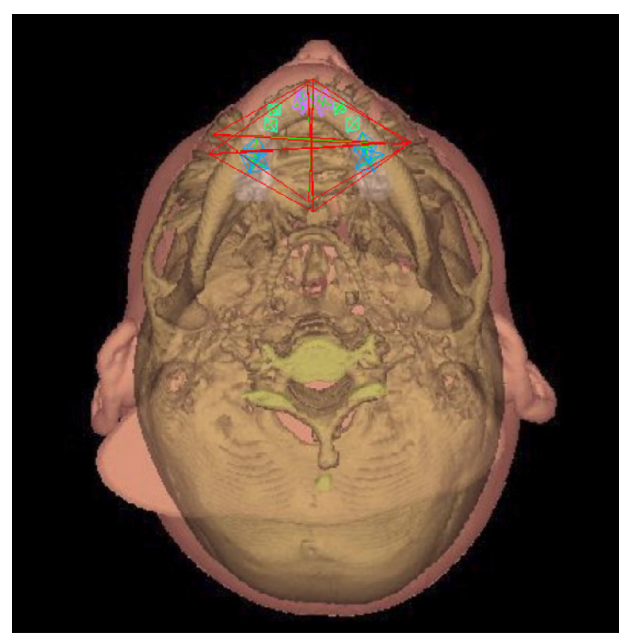

a

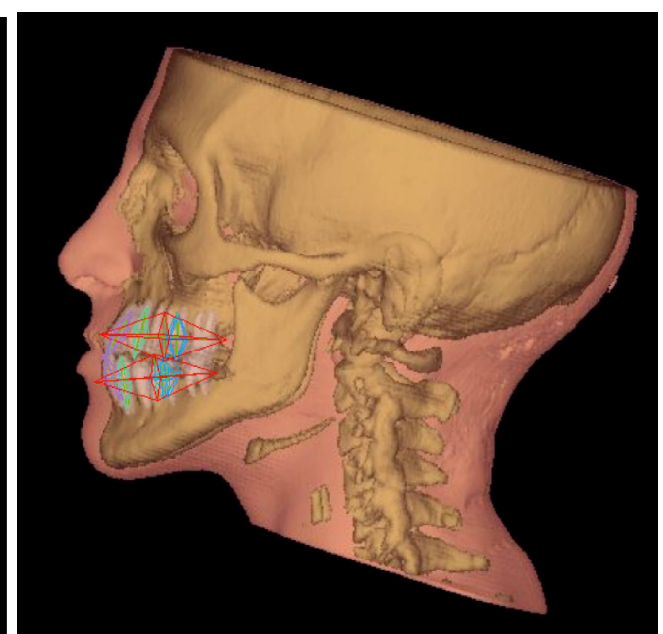

b

Figure 15

Analyse Cepha3DT en vues basale (a) et latérale gauche (b) : les enveloppes d'inertie montrent le décentrage des arcades en sens opposé.

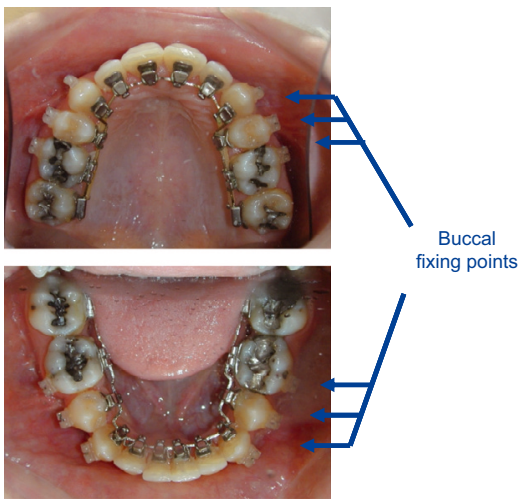

Figure 16

Fin de préparation orthodontique : des plots en céramiques sont collés avant la chirurgie. Les arcs sont en acier $.018 \times .025$ légèrement réduits sur les secteurs latéraux. 


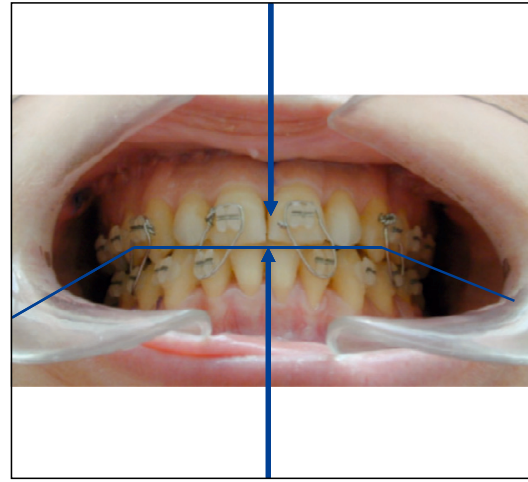

a

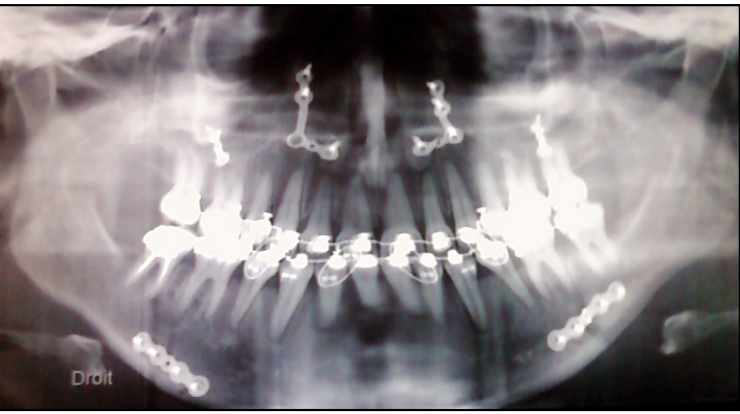

b

Figure 17

Blocage chirurgical assurant la symétrisation du plan d'occlusion et la concordance des milieux. (a) Vue endo-buccale (chirurgie, Dr Jean-François Baron), (b) orthopantomogramme.

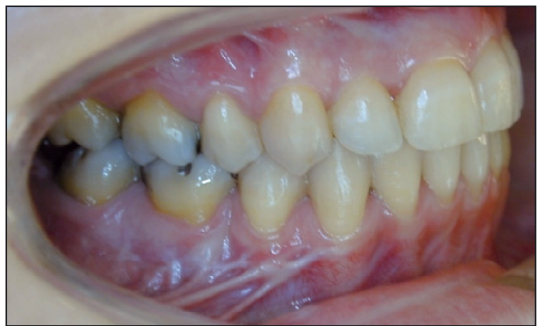

a

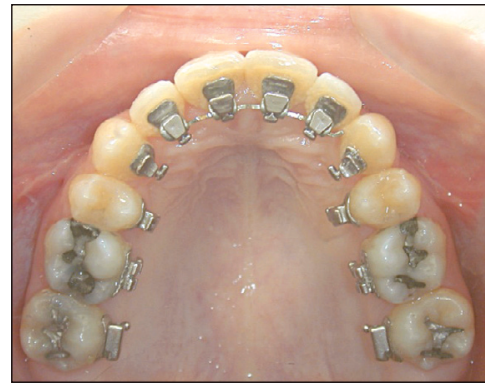

d

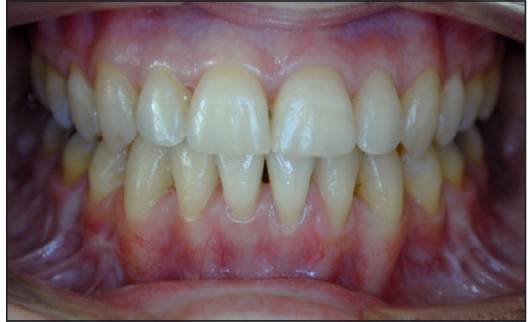

b

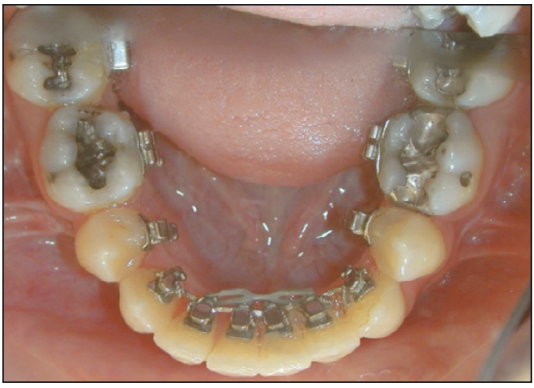

e

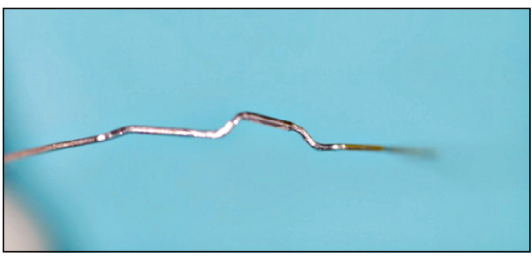

g

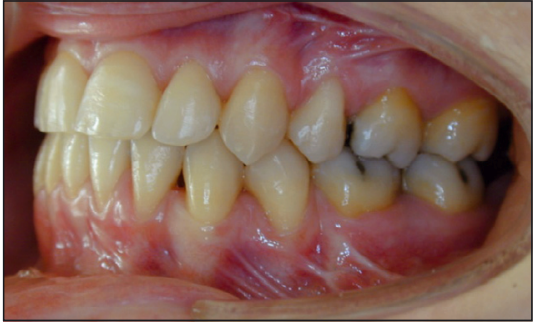

C

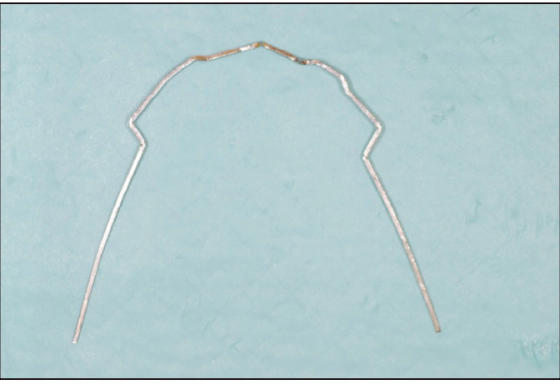

f

Figure 18

(a-e) Après 21 mois, intercuspidation/finitions, $(f, g)$ courbures de finitions sur l'arc terminal.

\subsection{2. $\operatorname{Cas} \mathrm{N}^{\circ} 2$}

Emma, 24 ans, est agent commercial et consulte pour aligner son bloc incisivo-canin supérieur qui disgracie son sourire. Elle ne peut porter qu'un appareil esthétique qui doit avoir le moins d'incidence possible sur son travail.
La face est équilibrée, le profil orthofrontal. Les relations occlusales sont en classe I. L'encombrement incisivo-canin est de $5 \mathrm{~mm}$ aux deux arcades (Fig. 22 et 23). Nos objectifs thérapeutiques sont l'alignement des arcades et leur parfaite intercuspidation, ainsi que le maintien des relations squelettiques et dento-squelettiques. 


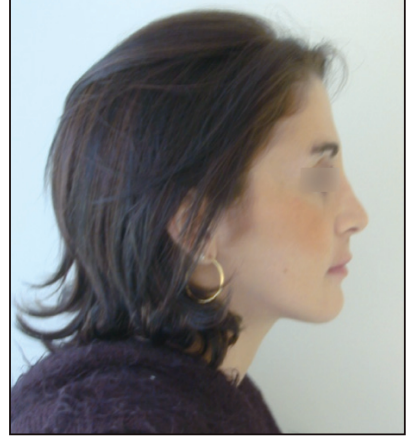

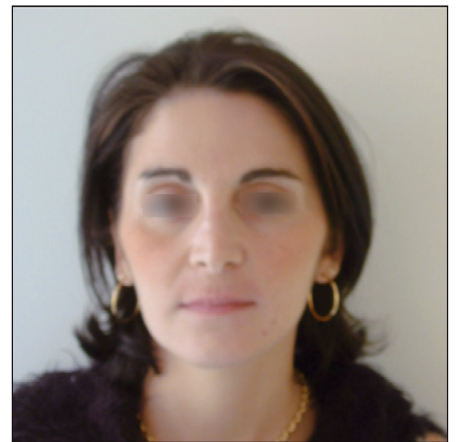

b

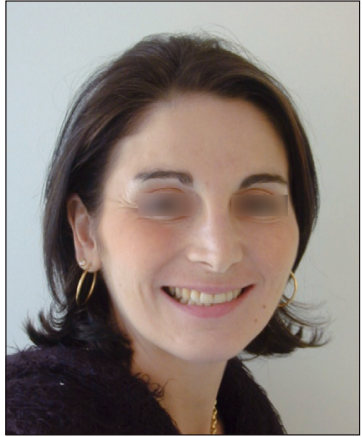

C

Figure 19

Vues exo-buccales après traitement.

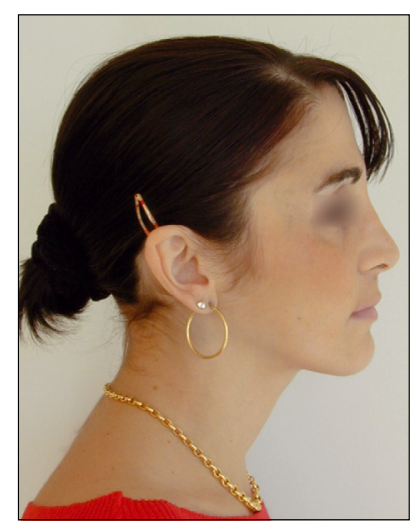

a

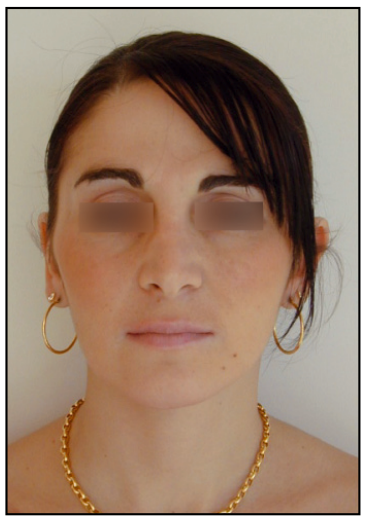

b

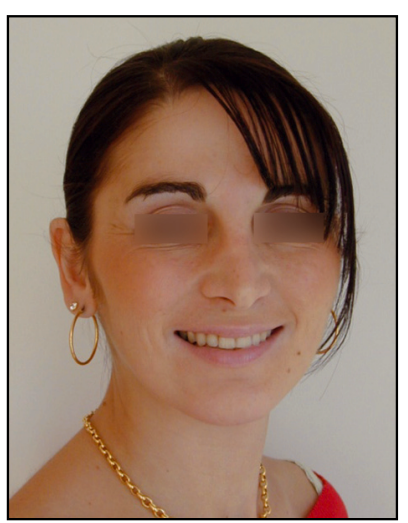

c

Figure 20

Vues exo-buccales quatre ans après le traitement.

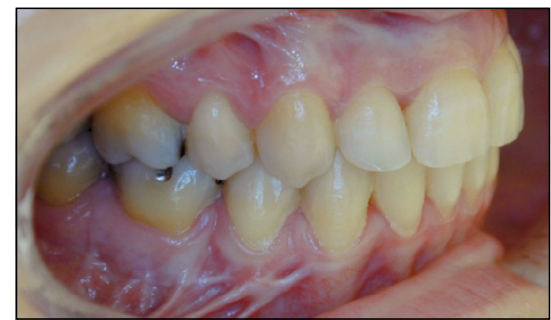

a

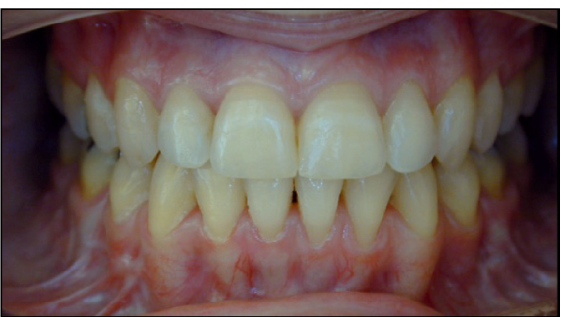

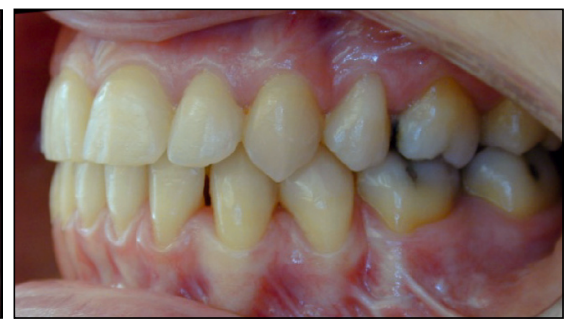

Figure 21

L'occlusion est bien calée; sans contention, le résultat à long terme reste stable.

L'indication d'une réduction amélaire interproximale étant écartée, le plan de traitement prévoit alors l'extraction des $15,25,35,45$, le recul prémolocanin, l'alignement et la fermeture des espaces résiduels aux deux arcades.

Le système utilisé est ici une technique « customisée » d'arc droit qui va permettre d'allier flexibilité et fiabilité. Larcade supérieure est d'abord appareillée avant l'extraction des 15 et 25 , le collage sur la 22 est différé et un premier arc en NiTi .016 est engagé
(Fig. 24). Le recul prémolaire et canin est effectué avec des accessoires de perte d'ancrage réciproque utilisant les crochets des brackets; l'absence d'in-set prémolaire sur l'arc droit permet un glissement rapide et sans friction (Fig. 25). Lorsque la place est disponible sur l'arcade, la 22 est collée sans difficulté en technique directe grâce à sa base individualisée (Fig. 26).

À l'arcade mandibulaire, l'arcade est collée «by pass » 33, 41 et un arc droit .014 NiTi est mis en 


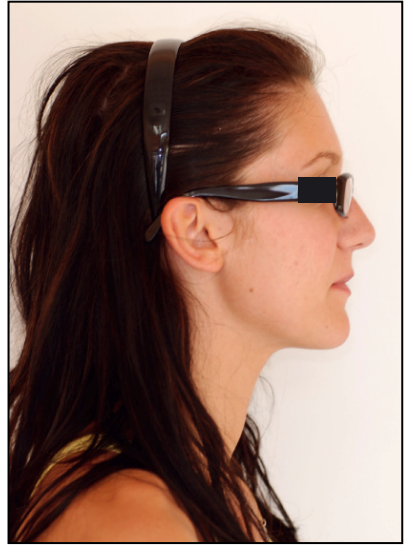

a

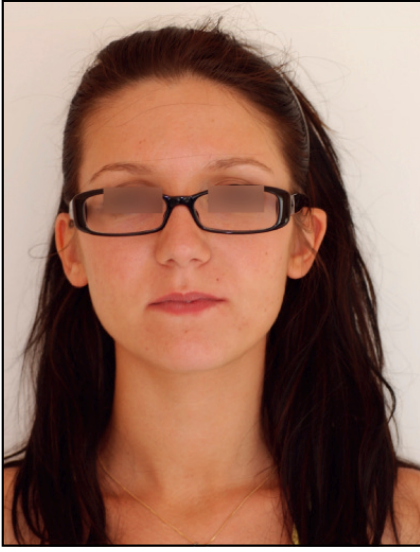

b

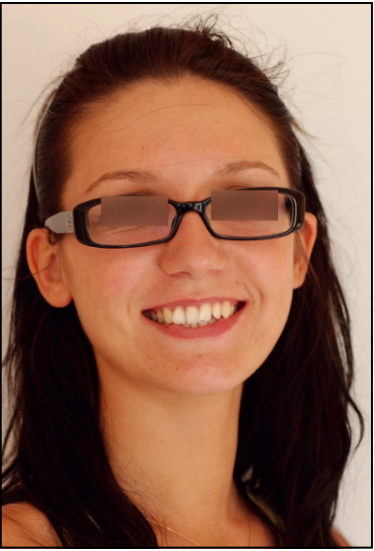

C

Figure 22

Vues exo-buccales : la face est harmonieuse, équilibrée.

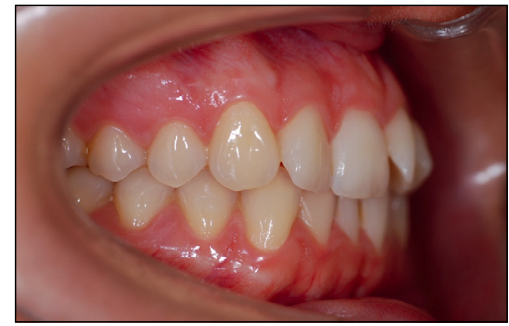

a

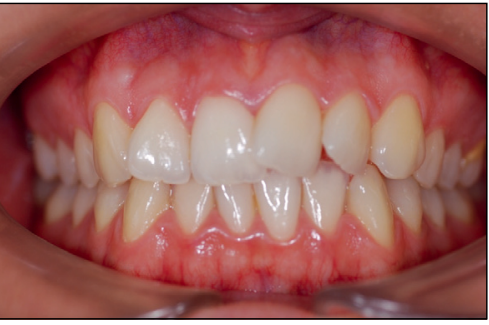

b

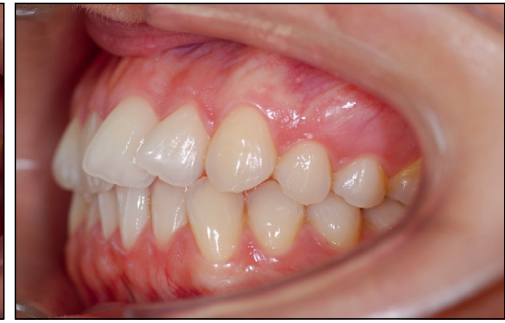

C

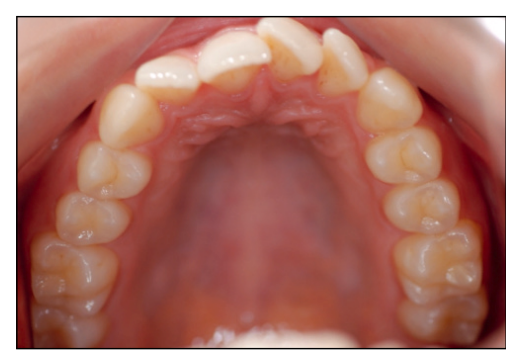

d

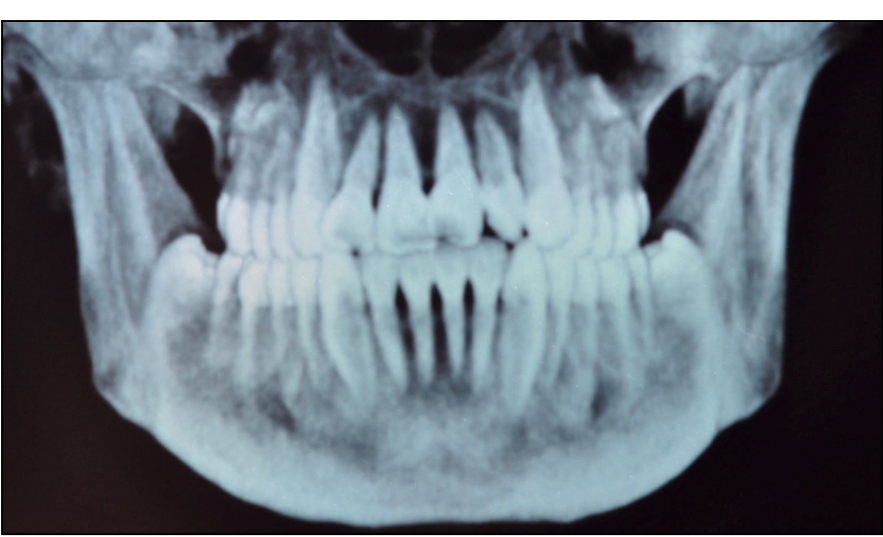

$f$

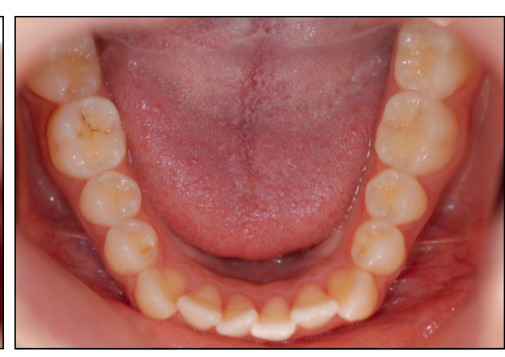

e

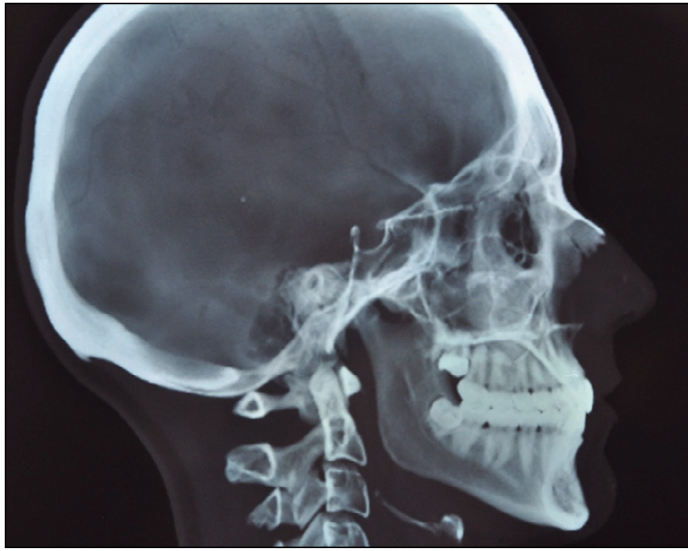

g

Figure 23

(a à e) vues endo-buccales : encombrement aux deux arcades, (f et g) imagerie avant traitement. 


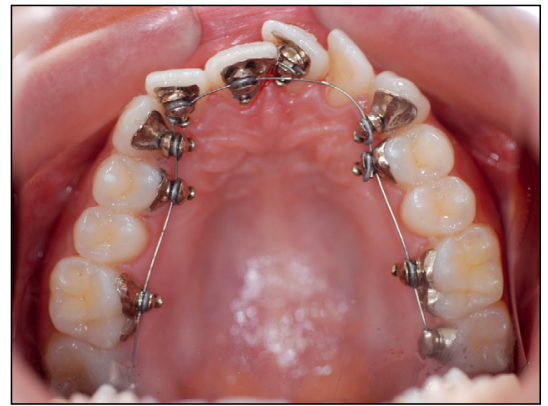

Figure 24

Premier arc maxillaire.

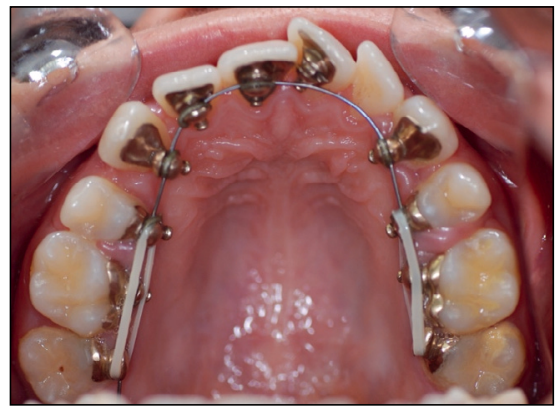

Figure 25

Distalisation prémolaire et canine.

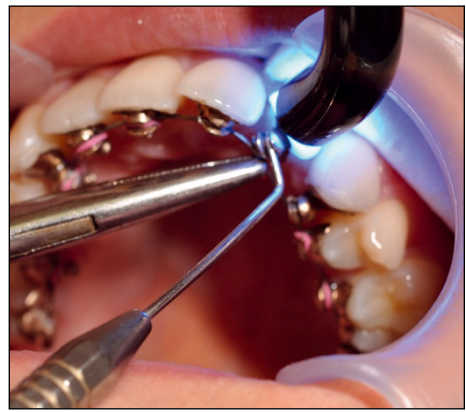

Figure 26

Collage de la 22 en méthode directe.

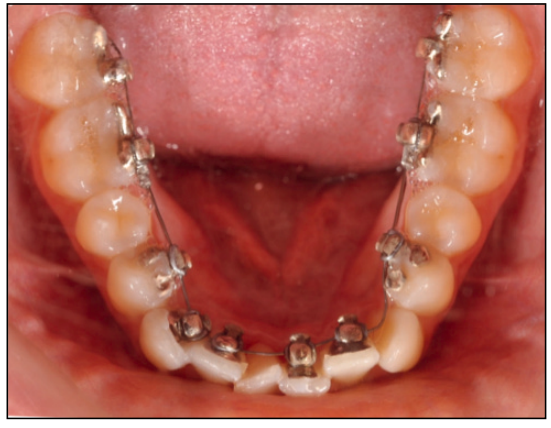

a

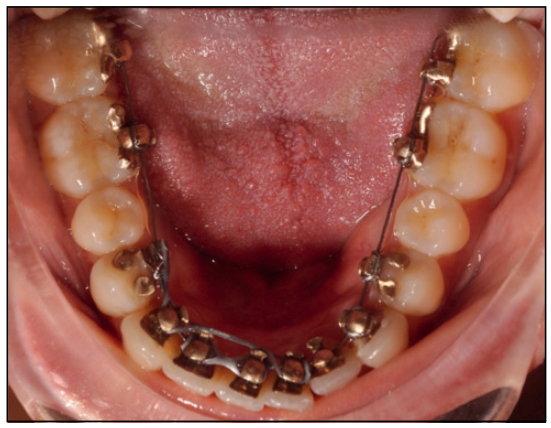

C

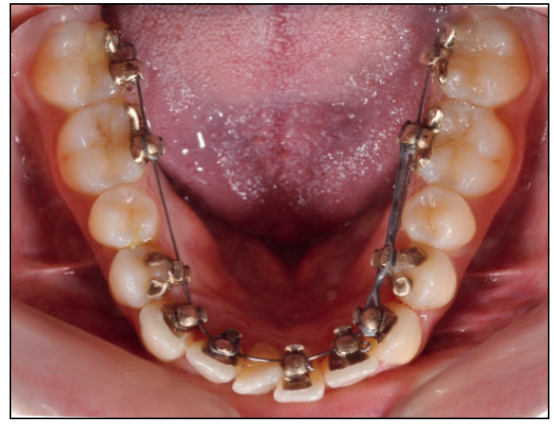

b

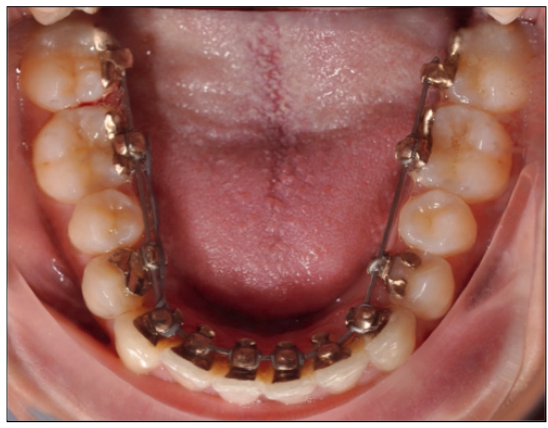

$\mathrm{d}$

Figure 27

Alignement progressif par prise en charge séquentielle et augmentation des sections d'arc droit.

place. Le collage séquentiel se poursuit à mesure que s'opère le nivellement et jusqu'à obtenir une arcade alignée et nivelée (Fig. 27) par un arc .017 ×.025 NiTi. A ce stade, l'absence de dysharmonie interarcade et une occlusion des secteurs latéraux compatible avec une bonne intercuspidation nous font renoncer à l'indication d'extraction et demander la fabrication en CFAO des brackets de 35 et 45 .

Après stabilisation par des arcs rectangulaires $.017 \times .025$ et $.018 \times .018$, les finitions sont entreprises. Le pliage minutieux dans les trois ordres si nécessaire, permettant de parfaire l'occlusion et l'esthétique finale, peut se faire beaucoup plus librement sur un arc droit, dépourvu d'information et de plicatures d'origine. Des élastiques verticaux sont utilisés (Fig. 28).

À l'issue de 19 mois de traitement, le résultat donne une classe II thérapeutique occlusalement équilibrée; le sourire est restauré. Les dents de sagesse supérieures ont fait leur évolution en bouche et seront conservées. Des contentions provisoires, $1 / 1$ et 3/3 sont collées (Fig. 29). Les radiographies 


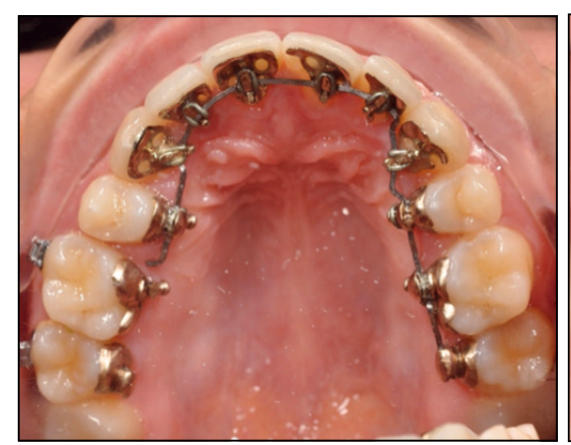

a

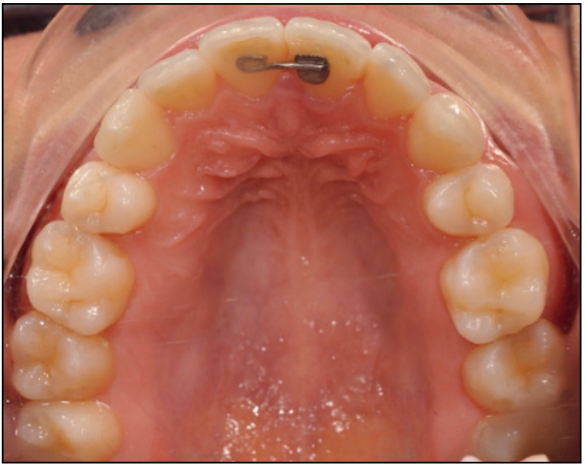

a

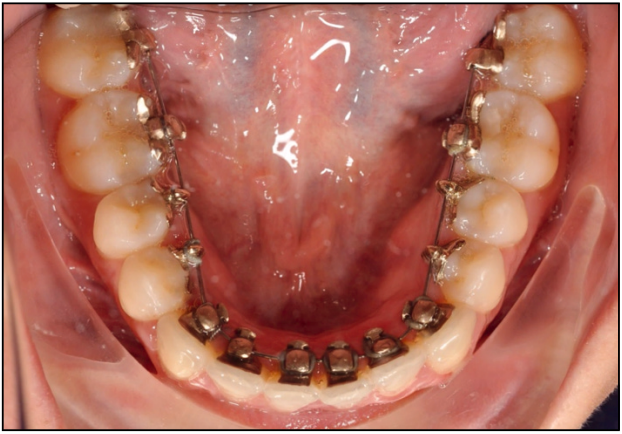

b

Figure 28

Finitions à la pince et aux élastiques.

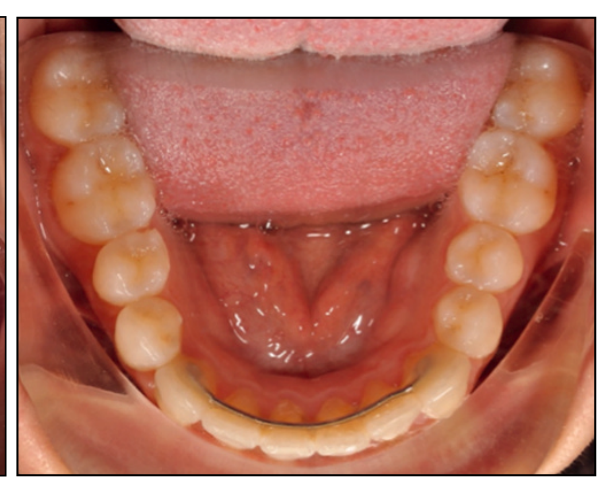

b

Figure 29

Contentions collées sur 11, 21 et 33, 43.

de fin de traitement montrent des rapports dentosquelettiques normaux (Fig. 30). Les objectifs de traitement ont été respectés (Fig. 31 et 32).

La présentation de ces cas cliniques traités avec deux types d'appareils montrent la mise en place de solutions techniques spécifiques propres à chaque dispositif. Le praticien doit en connaître les avantages autant que les limites afin d'atteindre ses objectifs de traitement et de choisir des options que lui seul détermine. Utiliser plusieurs techniques en même temps offre théoriquement l'intérêt d'augmenter son panel thérapeutique. N'en retenir qu'une seule permet de systématiser sa pratique et génère un gain de temps et d'efficacité qui sont les deux éléments récurrents de ce type d'orthodontie.

\subsection{Conclusions sur l'orthodontie linguale}

Que pouvons nous retenir des techniques linguales en tant que traitement invisible?

Si l'on s'en tient à cette seule exigence, elles remplissent parfaitement leur rôle. Cependant, malgré les progrès techniques, elles demandent encore une grande concentration de la part des praticiens à tout moment de la prise en charge thérapeutique. Pour le patient, elles demandent des concessions particulières par rapport à d'autres moyens de traitement.

Pendant longtemps, l'orthodontie linguale s'est retranchée derrière des barrières thérapeutiques pour justifier des compromis de résultat s'autorisant trop souvent des choix d'extractions «non conventionnelles » ou de non-extractions abusives, des quantités de stripping exagérées, des finitions décevantes ou des "condamnations à la contention à perpétuité ». Cela n'est plus valable aujourd'hui si tant est que ce le fût un jour. Avec l'affinement des techniques et du matériel, le développement de sociétés scientifiques telles l'ESLO (European Society of Lingual Orthodontics) ou le WSLO (World Society of Lingual Orthodontics), la création d'un board mondial, les exigences en qualité de traitement se sont nettement accrues et les cas présentés s'élèvent aux plus hauts standards de qualité. 


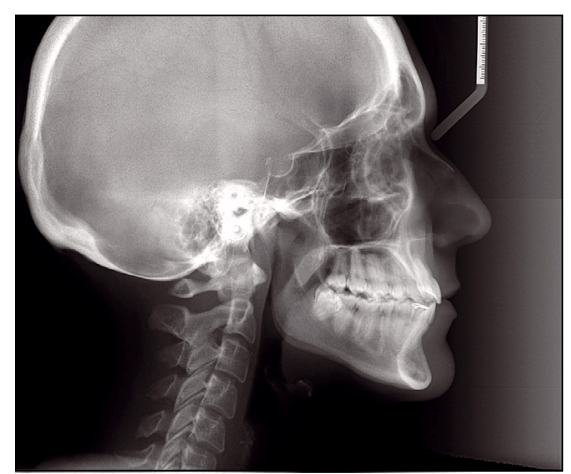

a

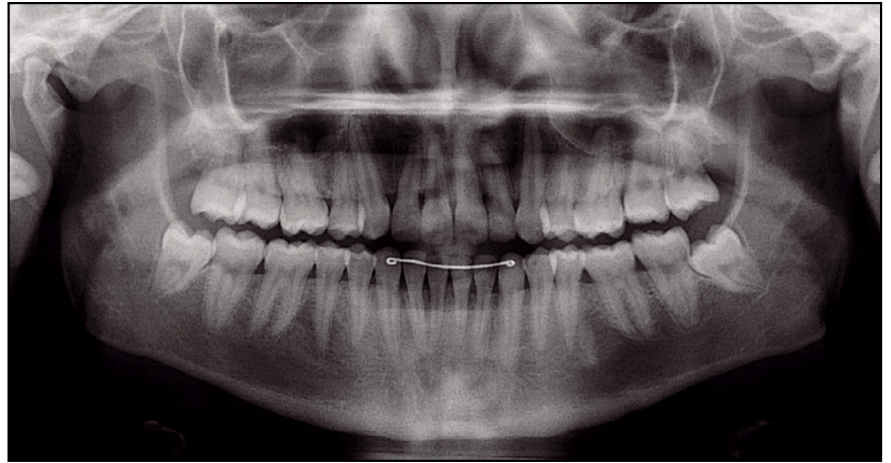

b

Figure 30

Radiographies post-thérapeutiques avec un pronostic de conservation des dents de sagesse favorable.

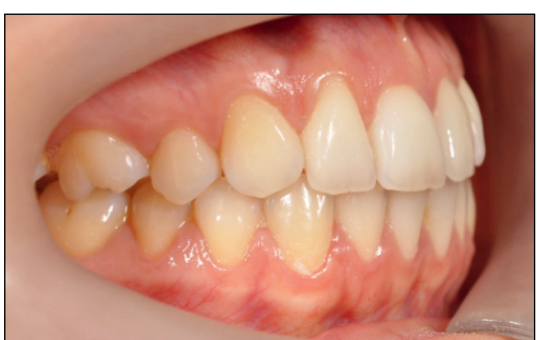

a

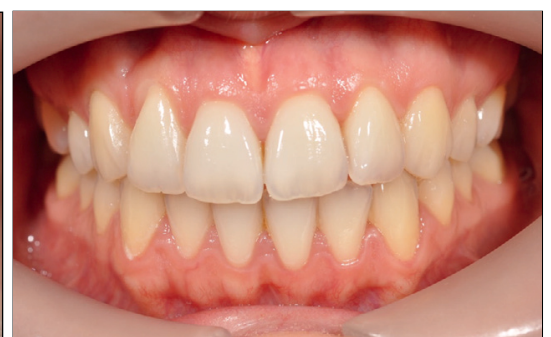

b

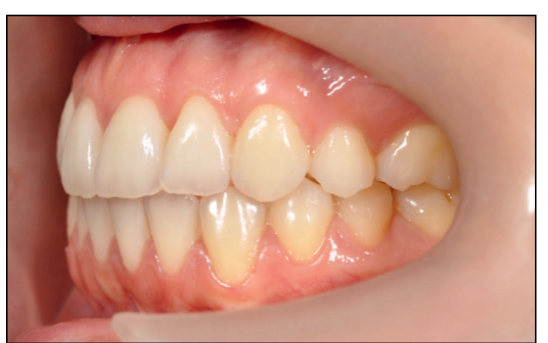

Figure 31

Intercuspidation de fin de traitement en classe II molaire thérapeutique.

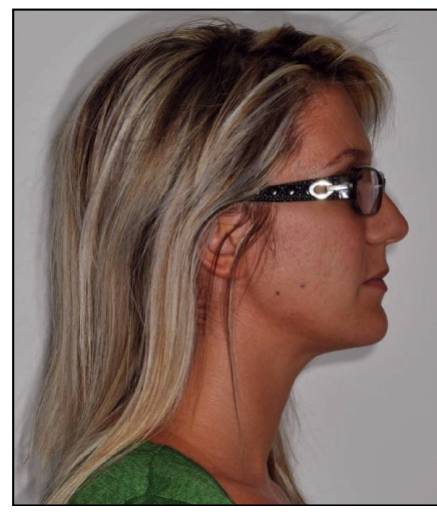

a

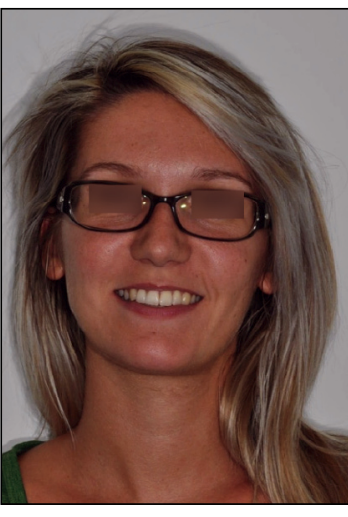

b

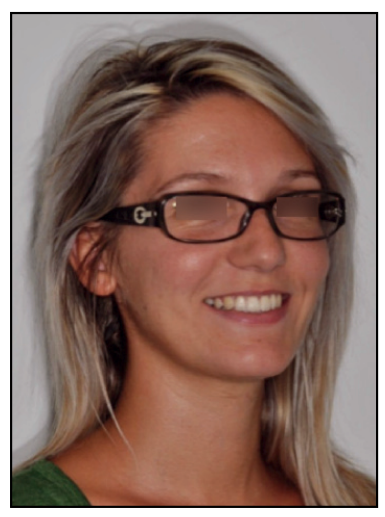

Figure 32

Le profil initial est restitué (a) et le sourire, vu de face (b) et de $3 / 4$ (c), est restauré.

Des cliniciens expérimentés ont mis en valeur, à partir de cas traités, l'efficacité des systèmes techniques qu'ils emploient comme Fillion [38], Scuzzo [109], Wiechmann [129], Cacciafiesta [21], Galletti [44], Lombardo [79], Mujagic [93], Takemoto [119], Becker [12], Baron [8], Muller [94] et bien d'autres. Certains tels
Inami [63], Fukawa [43], Hiro [55] ont donné à la technique linguale des lettres de noblesse supplémentaires en publiant des cas sévères avec des résultats exceptionnels parfaitement en accord avec les objectifs de normalité fonctionnelle, d'harmonie esthétique et de stabilité que l'on est en mesure d'attendre d'une technique universelle. Nous laissons 
la conclusion de cette partie à Toshiaki Hiro qui considère depuis toujours que "tous les cas qui peuvent être traités en orthodontie vestibulaire peuvent être traités en orthodontie linguale ».

\section{Lorthodontie presque invisible: les aligneurs transparents}

Lidée originale de cette nouvelle façon de déplacer les dents n'est pas neuve et a été émise et expérimentée par Harold Kesling [68] dès 1945. La réalisation de set-up et la fabrication consécutive de "tooth positioner » en caoutchouc ont permis de réaliser les premiers traitements par gouttière. Il prévoyait, dès que la technologie des matériaux le permettrait, que les traitements d'orthodontie pourraient être entièrement faits selon ce procédé. Revenu au goût du jour avec la création d'Invisalign, de nombreuses publications ont pu apprécier les avantages et les inconvénients des traitements réalisés par une série de gouttières thermoformées baptisées «aligners » (aligneurs) dans la presse professionnelle ou grand public. Le premier article de référence est publié en 2000 par Boyd, et al. [15]. S'agissant d'une pratique inconnue auparavant, les études s'intéressent aux bases et aux principes de la technique $[72,85,132]$. En dehors des rapports de cas traités, les investigations ont porté sur les effets thérapeutiques, les matériaux de fabrication, la mécanique, la biocompatibilité. Les appréciations cliniques ont évolué dans le temps. A sa sortie, le système Invisalign était réputé pouvoir traiter des cas de difficulté faible à moyenne sans extractions, des encombrements ou fermetures d'espaces jusqu'à $5 \mathrm{~mm}$, sans correction intermaxillaire. Certains utilisateurs [16, 90, 130] l'appliquent à des préparations chirurgicales ou pour corriger des béances légères voire des articulés latéraux inversés. Une étude sur cas traités $[14,27]$ conclut à une efficacité du procédé dans les cas d'alignement antérieur, de supraclusion et de décalages transversaux. En 2005, une revue systématique de littérature [77] sur Invisalign ne retient seulement que deux études cliniques [14,29] remplissant les critères d'inclusion avec au total très peu d'enseignements et un très pauvre niveau de preuve. De nombreux cliniciens ont continué à présenter des recherches et des cas traités en mettant en exergue les possibilités de corriger des cas complexes avec ou sans extractions. La deuxième revue systématique [18], parue en 2011, est un peu plus riche avec 23 articles retenus comprenant, entre autres, des essais randomisés et des études de cohortes. Cependant, les conclusions sont encore décevantes et sans évidence scientifique et suggèrent que des études comparatives sur l'efficacité thérapeutique sont nécessaires. Un travail d'analyse bibliographique récent [76], sur la période 2000 à 2011, conclut que cette technique convient aux besoins esthétiques et fonctionnels des patients dans la plupart des cas. Aujourd'hui, le nombre des travaux ne manque pas. Fin 2013, si on lance sur le moteur de recherche de l'EBSCO, une recherche avec en mots-clés «Invisalign » et/ou «clear aligner », on obtient pas moins de 274 résultats dont 109 publiés dans des journaux à comité de lecture. Lintérêt croissant du recours par les praticiens à ce type de solution peut s'apprécier par le volume d'articles et de présentations dans les différentes revues. L'hétérogénéité des formats d'étude, des objectifs, des méthodes ne permet pas pour le moment, d'éclairer le débat sur la validité de la technique.

\subsection{Caractéristiques techniques des systèmes d'aligneur}

En parallèle à cet intérêt croissant dans la littérature, des apports techniques ont été incorporés à la version initiale par Align Technology : des taquets accessoires collés avec une géométrie adaptée selon les mouvements à contrôler, des options de découpe permettant de coller des attaches ou d'attacher des élastiques, des reliefs améliorant l'effet de pression au collet, le changement du matériau de meilleure élasticité constituent des améliorations du procédé. Des versions spécifiques ont été mises sur le marché à destination des traitements courts, sectoriels, pour adolescents avec évolution de denture, d'alignement léger, de contention avec les versions Lite, Anterior, Teen, i7, Vivera permettant d'étendre la gamme de proposition thérapeutique avec des offres de prix correspondantes.

À côté de cela, d'autres fabricants ont emboîté le pas et sont apparus, parmi les principaux challengers, Clear Aligner (appellation partagée par plusieurs fabricants - Dr Tae Weon Kim - SheuDental - CA Laboratories Ltd...), Orthocaps (Ortho Caps GmbH, Hamm, Allemagne), Insignia ClearGuide Express (Ormco Corporation, Orange, CA), ClearCorrect (ClearCorrect LLC, Round Rock, TX), 
Tableau 1

Caractéristiques des principales marques d'aligneurs.

\begin{tabular}{|c|c|c|c|c|}
\hline $\begin{array}{l}\text { Nom commercial/ } \\
\text { Fabricant }\end{array}$ & $\begin{array}{l}\text { Type de gouttières/ } \\
\text { durée }\end{array}$ & Spécificités & $\begin{array}{l}\text { Modèles/ } \\
\text { \# de gouttières }\end{array}$ & $\begin{array}{l}\text { Indications/ } \\
\text { malocclusions }\end{array}$ \\
\hline $\begin{array}{l}\text { TwinAligner/ } \\
\text { Ortho Caps }\end{array}$ & $\begin{array}{l}\text { Souple (nocturne) } \\
+ \text { Dure (diurne)/3sem. } \\
\text { - par série de } \\
10 \text { gouttières }\end{array}$ & $\begin{array}{l}\text { - Friction pads/ } \\
\text { contrôle 3D } \\
\text { (>janv. 2014) }\end{array}$ & $\begin{array}{l}\text { Bi-arcade/illimité } \\
\text { Mono-arcade/illimité } \\
6 \text { antérieures/illimité }\end{array}$ & $\begin{array}{l}\text { Toutes } \\
\text { Monomaxillaire } \\
\text { Incisivo-canine }\end{array}$ \\
\hline $\begin{array}{l}\text { Invisalign/ } \\
\text { Align } \\
\text { Technology }\end{array}$ & $\begin{array}{l}\text { Dure/15 jrs } \\
\text { - Possibilité } \\
\text { de réajustement } \\
\text { possible en cours } \\
\text { de traitement }\end{array}$ & $\begin{array}{l}\text { - Optimized attachements/ } \\
\text { contrôle unitaire 3D } \\
\text { - Power ridges/torque ant. } \\
\text { - Deep Bite attachements/ } \\
\text { ingression incisive } \\
\text { (> fev.2014) }\end{array}$ & $\begin{array}{l}\text { Full/illimité } \\
\text { Teen/Illimité } \\
\text { Lite/N=14 } \\
\text { i7/N=7 } \\
\text { Vivera/N=3 }\end{array}$ & $\begin{array}{l}\text { Toutes } \\
\text { Denture mixte } \\
\text { modérées } \\
\text { légères } \\
\text { Contention }\end{array}$ \\
\hline $\begin{array}{l}\text { Insigna } \\
\text { ClearGuide } \\
\text { Express/Ormco }\end{array}$ & $\begin{array}{l}\text { Lisse/15 jrs } \\
\text { - réévaluation par } \\
\text { " mordu " } \\
\text { à mi-traitement }\end{array}$ & - & $\begin{array}{l}\text { ClearGuide Express/ } \\
\mathrm{N}=20 \\
\text { Simply } 5 / \mathrm{N}=10 \\
\text { - mono ou bi-arcade }\end{array}$ & $\begin{array}{l}\text { Toutes antérieures } \\
\text { Légères antérieures }\end{array}$ \\
\hline $\begin{array}{l}\text { Cristal Aligneur/ } \\
\text { Cristal } \\
\text { Orthodontie }\end{array}$ & $\begin{array}{l}\text { Dure/variable + Fine/ } \\
\text { variable - seulement } \\
\text { pour modèle cristal }\end{array}$ & - & $\begin{array}{l}\text { Cristal/N=24 } \\
\text { Jade/N=14 } \\
\text { Ambre/N=6 }\end{array}$ & $\begin{array}{l}\text { Toutes } \\
\text { Modérée } \\
\text { Légère }\end{array}$ \\
\hline $\begin{array}{l}\text { Clear Aligner } \\
\text { (Dr TW Kim)/ } \\
\text { Sheu-Dental }\end{array}$ & $\begin{array}{l}\text { Fine/ } 15 \text { jrs } \\
\text { Medium/15 jrs } \\
\text { Dure/15 jrs } \\
\text { - par série de } 2 \text { jeux }\end{array}$ & - & $\begin{array}{l}\text { Unitairement ou par } \\
\text { groupe de dents } \\
\text { selon prescription }\end{array}$ & Légères \\
\hline
\end{tabular}

MTM Clear Aligners (Dentsply Int, York, PA), Cristal Aligner (CristalOrthodontie, Casablanca, Maroc)... Des différences techniques existent entre ces divers produits (Tab. 1).

Les systèmes d'aligneurs ont cependant des points communs :

- Lacquisition numérique de la denture du patient pour créer un modèle virtuel 3D : ceci est réalisable par un scanner des modèles en plâtre, par un scanner des empreintes PVS, ou directement à l'aide d'un scanner intra-oral. La plupart des fabricants acceptent les fichiers 3D avec des conditions de compatibilité plus ou moins restrictives. Par exemple, Invisalign n'accepte que des empreintes numériques saisies avec la caméra iTero (Fig. 33).

- Lutilisation d'une interface logicielle présentant sur écran les dents en occlusion terminale (Fig. 34 et 35): le praticien peut intervenir de façon directe ou non pour modifier le résultat. Il peut, selon le degré de sophistication du programme, suivre la progression de la correction étape par étape et disposer de superpositions 3D début/fin.
- Lincorporation des prescriptions thérapeutiques qui offrent à l'orthodontiste une certaine convivialité dans la conduite du traitement : il peut incorporer des informations de sur-correction, ainsi que des accessoires de traitement, attaches, boutons, découpes, ainsi que modifier les options thérapeutiques, stripping, mécanique intermaxillaire, chirurgie, expansion...

- La réalisation matérielle de séries de gouttières qui sont fabriquées à l'avance selon une planification virtuelle des mouvements désirés: le nombre de gouttières est fonction de la complexité et de l'importance des déplacements dentaires programmés.

- Lutilisation de matériaux transparents thermoformables, gage d'acceptabilité par le patient : l'esthétique est conditionnée par la translucidité du matériau et le confort particulier vient du caractère amovible de l'appareil. Pour chaque aligneur, des modèles physiques sont fabriqués par des procédés de CFAO sur lesquels sont emboutis des plaques résines qui sont ensuite découpées au collet pour constituer un aligneur. 


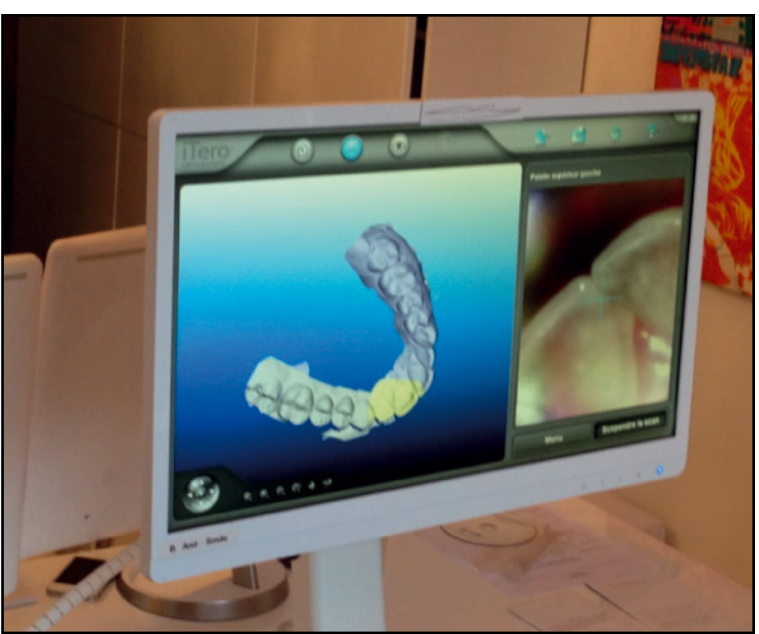

a

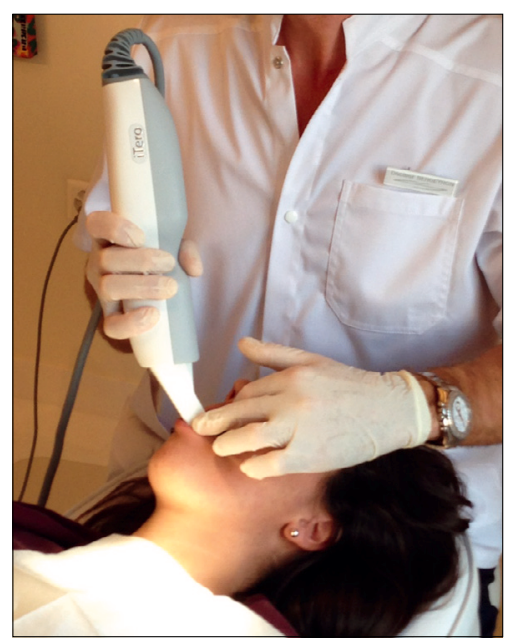

b

Figure 33

Saisie des empreintes numériques par scanner intra-oral iTero ${ }^{\circledR}$ (Dr Patrice Bergeyron).

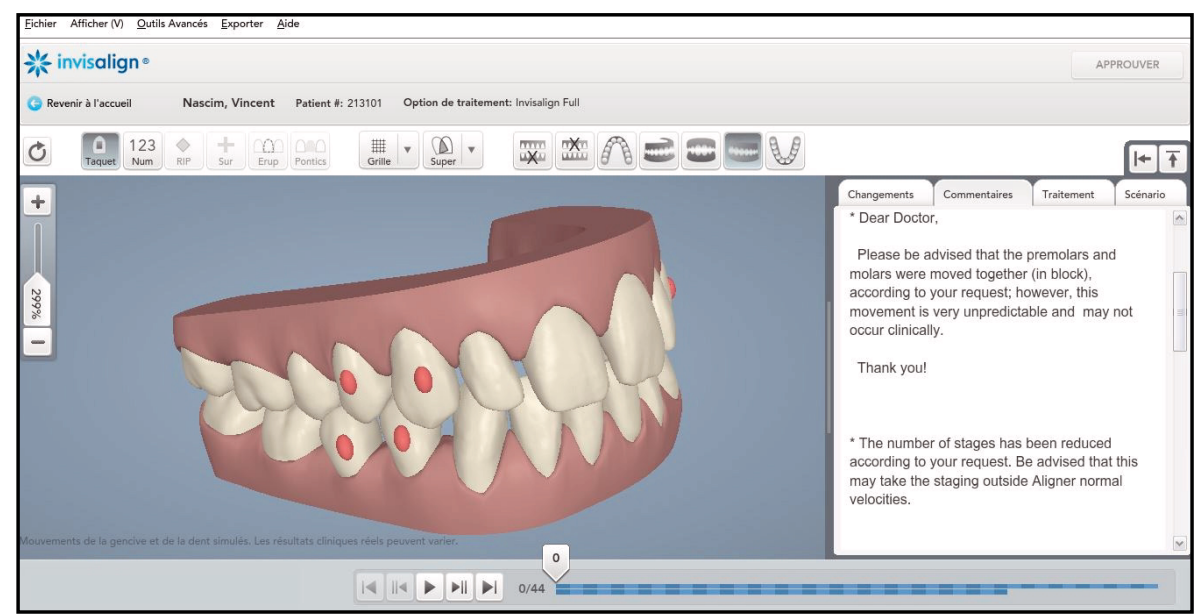

Figure 34

Environnement logiciel du Clin Check ${ }^{\circledR}$ (Invisalign ${ }^{\circledR}$ ) qui permet la conception du traitement virtuel.

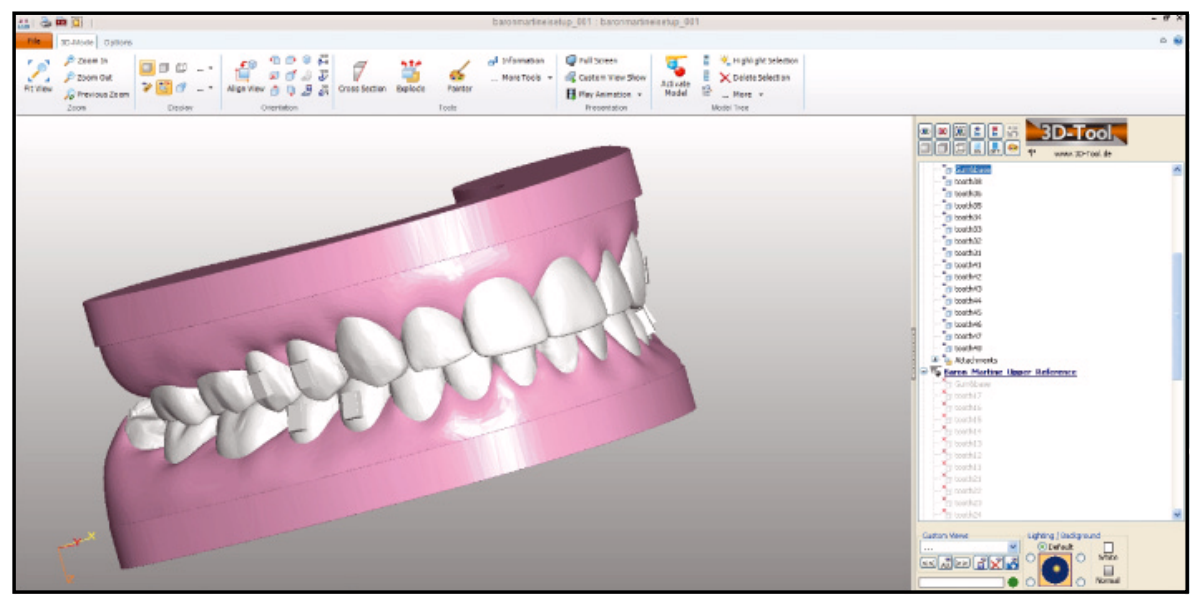

Figure 35

Environnement logiciel i-Set-up (Dr Orthocaps ${ }^{\circledR}$ ). 


\subsection{Potentialité des techniques d'aligneurs}

Plusieurs questions restent posées avant d'envisager la généralisation de ce type d'orthodontie.

- Les conditions biomécaniques : les forces délivrées aux dents sont produites par le retour à sa position neutre de la gouttière initialement introduite en force sur les dents. La quantité de déplacement est située selon les constructeurs entre $0,25 \mathrm{~mm}$ et $1 \mathrm{~mm}$. Elles dépendent donc des propriétés du matériau utilisé et du système de force délivré en tridimensionnel par la gouttière. La plupart des fabricants utilise des plaques thermoformées en résine polyuréthane ou en polypropylène dont les propriétés sont fonction des conditions de température, de l'humidité, de l'exposition aux milieux enzymatiques et de contraintes mécaniques $[65,73,107]$. Il a été montré que la dureté Vickers pouvait augmenter en milieu oral, ce qui peut s'expliquer par l'altération de la structure cristalline du matériau [34]. D'autres travaux [105] n'ont pas observé de modification de propriétés. Le rôle de l'épaisseur de la plaque a été mis en évidence aussi bien pour les gouttières dures que pour des gouttières souples [52,73]. Pour une même marque de plaque de thermoformage, une épaisseur de matériau plus fine délivre une énergie plus favorable. Les forces restituées sont également sensibles à la répétition de la mise en charge suggérant que les conditions de port des aligneurs ont un rôle à jouer. Align Technology a introduit avec la version G4 d'Invisalign un nouveau polymère censé délivrer une force plus constante sans décroissance d'intensité avec le temps, avoir une meilleure élasticité et permettre une insertion et une désinsertion plus facile des aligneurs. Une étude sur 1015 patients traités montre une amélioration significative du contrôle des mouvements de rotation et d'extrusion ${ }^{4}$ avec ce nouveau matériau. Les propriétés du matériau ne sont pas les seuls facteurs qui rentrent en jeu dans la bonne distribution des forces. Le mode de fabrication des gouttières utilise un procédé de stéréolithographie qui va générer pour chaque aligneur un modèle physique par apposition de couches successives donnant à l'échelle microscopique un profil en escalier. Ceci affecte la précision dimensionnelle $\mathrm{du}$ contour notamment pour les zones fortement convexes de la dent [23] et diminue l'efficacité de positionnement des aligneurs et la vitesse de dépla-

${ }^{4}$ www.aligntechinstitute.com/Products/SmartTrack/ cement des dents comme cela a été démontré pour le groupe incisif supérieur [83]. Le principe mécanique des aligneurs est relativement complexe et a fait l'objet de plusieurs études sur des gouttières souples [126] ou dures [52]. Les forces délivrées ont été mesurées sur une dent isolée ou sur un groupe de dents. Lintensité des composantes horizontale et verticale est jugée beaucoup trop élevée par rapport aux valeurs idéales [53]. Lépaisseur du matériau influe sur la valeur des forces permettant les mouvements de version et de torque. Par ailleurs, la durée d'application est maximale le premier jour de port, décroît à J2, puis reste stationnaire jusqu'à J15 [122]. Le contrôle du torque reste difficile. Pour une incisive, il résulte d'un couple de force s'exerçant au collet vestibulaire et au niveau de la face palatine du bord libre [4,20]. Pour qu'elle soit effective, il a été montré que la gouttière doit s'adapter intimement à la surface dentaire, ce qui est rendu difficile par une composante verticale qui a tendance à chasser l'appareil de la dent [53]. De ces différentes études sur la mécanique des aligneurs, outre le matériau utilisé et la précision du processus de fabrication, il apparaît que l'anatomie coronaire va jouer un grand rôle dans l'intensité et l'orientation des forces et couples de forces délivrés par des aligneurs avec, au total, une prédiction et un contrôle réduits quant à leurs effets. Une étude expérimentale animale montre qu'en dépit d'un mouvement recherché de mésialisation des molaires, les changements histologiques au niveau ligamentaire et osseux se sont traduits par une version distale et une ingression, associés à terme à des conditions de résorption radiculaire [115].

- La biocompatibilité

Suite à l'abrasion en bouche du matériau, le relargage de particules de polyéthylène est possible, pouvant entraîner une inflammation liée au port et au changement des aligneurs [107].

Des études de laboratoire sur des lignées cellulaires n'ont pas mis en évidence de cytotoxicité avérée ni de risque hormonal $[34,35]$ reliable à la présence de bisphénol A. La pointilleuse Food and Drugs Administration a approuvé le système Invisalign avant sa mise sur le marché et a récemment étendu son usage aux enfants en denture mixte et aux cas chirurgicaux. Une étude récente [102] s'est plutôt intéressée à l'isocyanate contenu dans le polymère et a testé in vitro sa neutralité biologique sur les kératocytes buccaux. Bien que des réactions cellulaires aient pu 
être observées par rapport au groupe de contrôle, aucune conclusion de nocivité ne peut être portée, appelant néanmoins à de nouvelles investigations.

Au-delà de l'avantage esthétique, un autre bénéfice pour le patient réside dans la facilitation de l'hygiène buccale grâce à l'aspect amovible de l'appareil. On assiste à une diminution des risques carieux et parodontaux $[86,87]$ vis-à-vis des appareils multi-attaches. Une comparaison sur un an de traitement [66] montre un meilleur état parodontal mesuré sur tous les indices parodontaux et les souches bactériennes pathogènes dans le groupe avec aligneurs. De plus, l'indice de plaque s'abaisse témoignant d'une amélioration de l'hygiène et de l'état parodontal au cours du traitement par gouttière, ce qui en fait une indication préférentielle dans les cas de parodontopathie.

- La prédictibilité et la reproductibilité des mouvements : les premiers travaux mesurant l'efficacité des aligneurs se sont évertués à déterminer le temps de port cliniquement idéal, le type de matériau et l'épaisseur les plus adaptés pour assurer les mouvements programmés [13,29]. Les indications ont été mieux cernées et préconisées. Les améliorations des dispositifs apportées par la suite et l'habileté des cliniciens ont permis d'élargir les possibilités thérapeutiques. De multiples travaux ont évalué l'efficacité de la réduction amélaire interproximale $[70,73]$, des attachements [70], des mécaniques de fermeture [32, $49,62,131]$ et réouverture d'espaces [100], ainsi que les préparations chirurgicales [15, 130]. Sur la grille de cotation de LBO, les cas traités en 2005 par Invisalign avaient un score de $27 \%$ inférieur aux autres cas [123]. Presque $80 \%$ des cas traités sélectionnés dans une autre étude [32] seraient refusés à la certification du Board contre 52 \% des cas traités en orthodontie conventionnelle. Plus récemment, un certain nombre d'études objectives ont remis en cause les propriétés essentielles que l'on peut légitimement attendre d'un appareil orthodontique. Des différences ont été objectivées entre déplacements prévus et déplacements effectués [32]. Seulement une efficacité moyenne de $41 \%$ a été mesurée avec des difficultés particulières pour l'égression (29,6 \%), la mésialisation et les rotations importantes. Il apparaît une nécessité de réévaluation avec des séries de gouttières successives afin d'améliorer les résultats. En 2013, selon la grille de cotation MGS (Model Grading System) utilisée par le Board américain, les principales faiblesses se situent, dans la réduction du surplomb, l'alignement des crêtes marginales, la qualité des points de contact inter-proximaux et la correction des rapports occlusaux [67].

\subsection{Discussion autour des avantages concurrentiels des aligneurs sur les autres systèmes orthodontiques}

Bien que l'efficacité thérapeutique soit toujours discutée, le nombre de professionnels utilisant la technique Invisalign ne cesse de croître. Align Technology affiche aujourd'hui plus de deux millions de cas traités. Un total de 40000 aligneurs sont fabriqués tous les jours dans ses usines mexicaines et on estime approximativement à 1,5 million le nombre de personnes qui portent les aligneurs de la marque. De plus, d'autres sociétés sont rentrées sur ce marché en expansion et participent à la généralisation et à la croissance de cette nouvelle forme d'orthodontie.

Les avantages pour le patient sont évidents : esthétique acceptable, confort, hygiène. De plus, l'appareil ne génère qu'une faible douleur à l'insertion qui disparaît en 2-3 jours $[88,96]$. Pour le praticien, il s'agit d'un autre exercice avec un temps au fauteuil fortement réduit, des possibilités d'organisation et de management autour du produit économiquement très porteur, un challenge pour maîtriser une technique à perfectionner.

Cependant, l'orthodontie par aligneurs présente des limites bien visibles. Car il ne s'agit pas de la transposition d'un système d'un côté à l'autre des dents, comme dans le cas de la technique linguale, mais de l'émergence d'une nouvelle forme de thérapeutique. D'un point de vue biologique, l'innocuité du système n'est pas encore définitivement établie alors que son utilisation est déjà planétaire. Même si quelques témoignages d'intolérance ou d'allergie sont rapportés sporadiquement par les internautes, il n'y a pour autant aucune preuve scientifique de toxicité [102]. Ceci doit être rapporté à la rareté et aux difficultés d'investigation sur ce sujet notamment par la réalisation d'études in vivo. Les conditions mécaniques sont fluctuantes d'un cas clinique à l'autre en fonction de la malocclusion initiale, de l'anatomie dentaire, de facteurs propres au patient. Initialement réservées à des corrections limitées, les indications sont toujours poussées un peu plus loin. Les résultats sont donc variables et leur prévision reste 


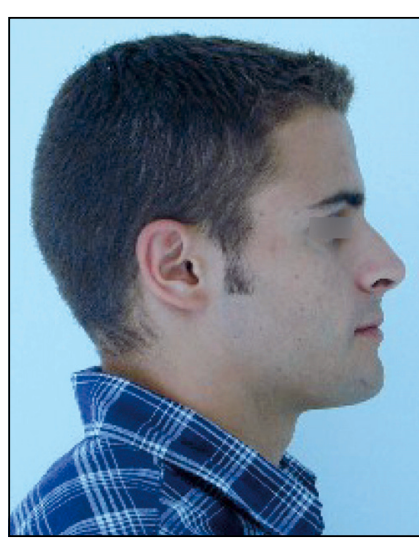

a

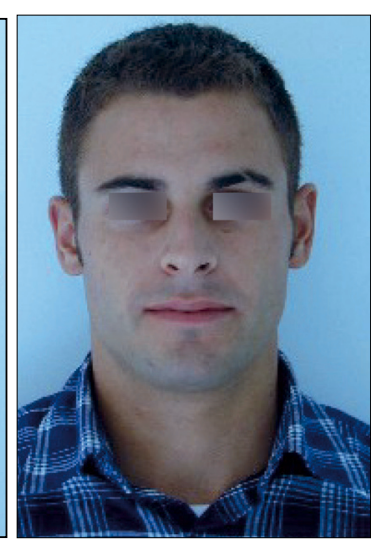

b

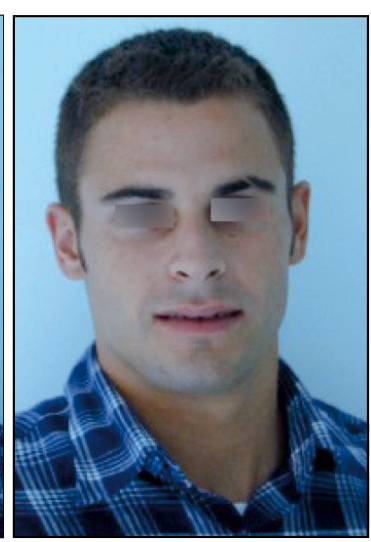

C

Figure 36

Vues exo-buccales pré-traitement.

délicate. À quelques exceptions près, présentant des canines en version particulièrement favorables, les cas avec extraction de prémolaires restent très difficiles à appréhender. Ici aussi, l'habileté du praticien et les exigences thérapeutiques qu'il s'impose sont des facteurs déterminants dans la qualité des résultats comme en témoignent certains cas publiés traités avec un grand savoir-faire [28].

Conscients des incertitudes, les fabricants misent également sur leur département de Recherche et Développement pour apporter des solutions techniques. Ainsi Invisalign ${ }^{\circledR}$ a vu son système évoluer en proposant des taquets à différentes géométries permettant un contrôle notamment en version, rotation et égression. Des accessoires de torque ainsi qu'un changement de matériau ont également apporté davantage de précision. Orthocaps ${ }^{\circledR}$ propose des surfaces de frictions spécifiques qui permettent une rétention multidirectionnelle de l'aligneur, ce qui, associé à un matériau élastique, accroît de façon substantielle la rétention de l'aligneur et donc génère des forces orthodontiques mieux conduites ${ }^{5}$. Insignia ClearGuide ${ }^{\circledR}$ propose un accès au setup virtuel qui va permettre au praticien lui-même d'anticiper et d'hypercorriger les déplacements. Enfin, des sociétés savantes dédiées aux aligneurs se constituent partout et réunissent des utilisateurs qui échangent leurs expériences et leurs travaux comme par exemple la SFOPA (Société Française

${ }^{5}$ www.zwp-online.info/de/zwpnews/

dental-news/branchenmeldungen/

innovation-2014-ortho-caps-stellt-neuerungen-vor
d'Orthodontie par Aligneurs) ou la DGAO (German Association for Aligner Orthodontics).

\subsection{Cas clinique}

Thomas a 23 ans et n'a bénéficié d'aucun traitement orthodontique avant de consulter pour des diastèmes aux deux arcades qui le gênent esthétiquement et occasionnent des rétentions alimentaires. Il est facialement équilibré, et son sourire est verticalement bien positionné. Les rapports d'occlusion se font en classe I bilatérale avec une infraclusion incisive de $0,5 \mathrm{~mm}$ et une légère proalvéolie incisive (Fig. 36, 37 et 38). On peut noter une dysharmonie dento-dentaire légère avec un indice d'harmonie esthétique incisive diminué en raison de l'insuffisance de taille des 12 et 22. Un traitement avec le système Invisalign est proposé pour résoudre sa malocclusion en 44 aligneurs. Les taquets sont collés sur les canines et prémolaires (Fig. 39 et 40). Les aligneurs sont portés $22 \mathrm{~h} /$ jour et changés tous les 15 jours. Au bout de 18 mois, le traitement arrive à son terme; les objectifs sont atteints. L'occlusion de fin de traitement présente une inocclusion des secteurs postérieurs. Pendant les trois premiers mois de la période de contention, la dernière gouttière est portée de nuit à l'arcade supérieure puis ajourée; un fil 0,7 mm est collé sur 33 et 43. À l'issue, l'occlusion des secteurs latéraux se retrouve en bon calage (Fig. 40 et 41).

Les rapports antérieurs et postérieurs sont conformes aux prévisions. Le sourire est retrouvé (Fig. 42). Neuf ans après, l'occlusion est parfaitement assise et stable, libre de contention 


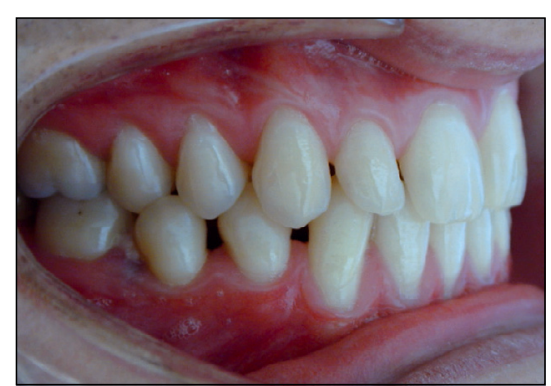

a

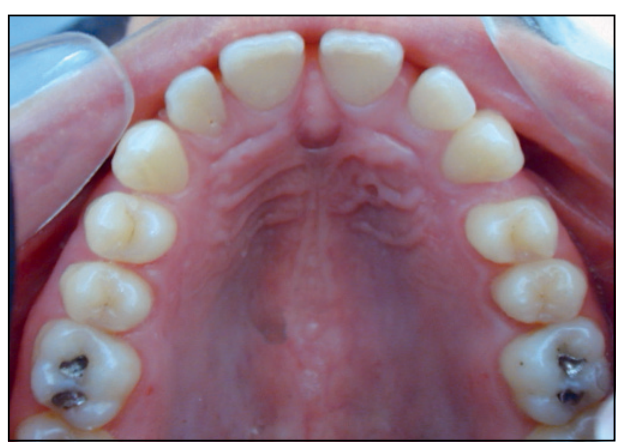

d

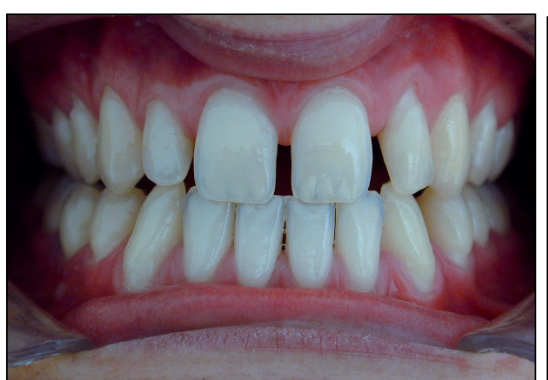

b

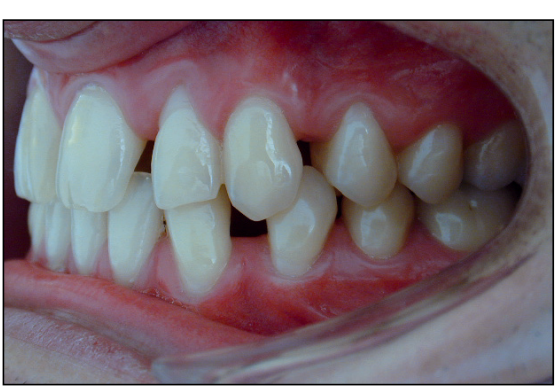

C

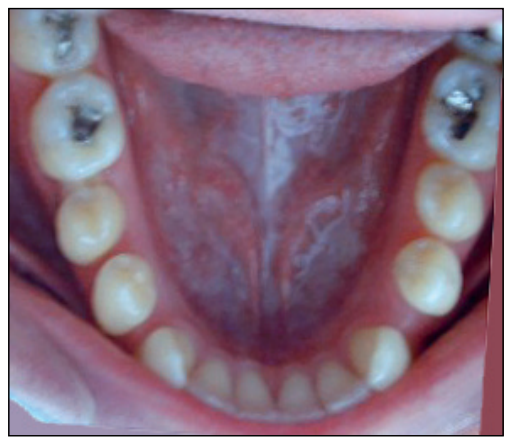

e

Figure 37

Vues endo-buccales pré-traitement.

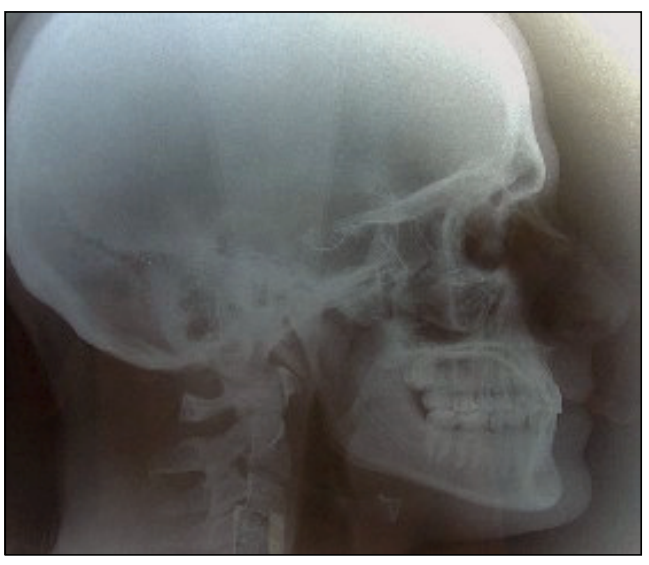

a

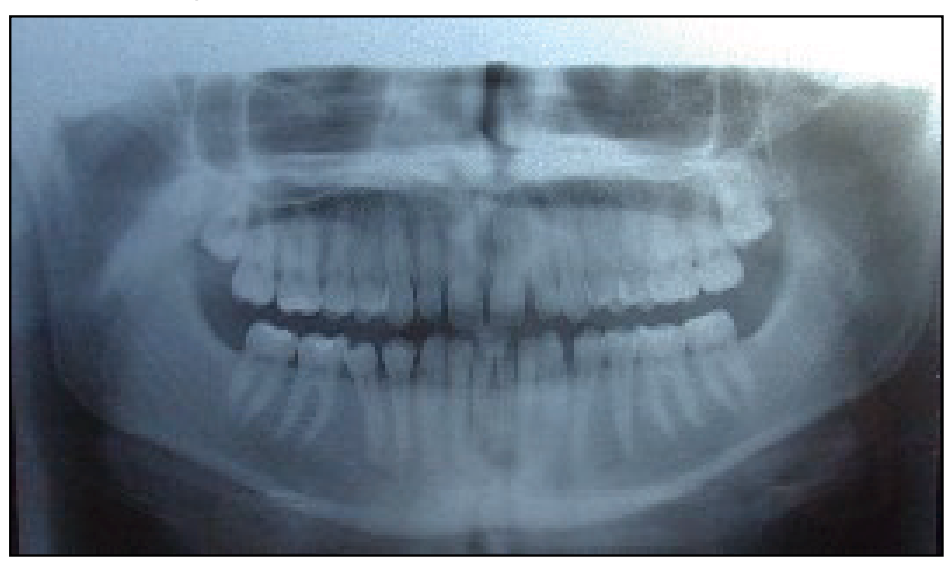

b

Figure 38

Radiographies avant traitement.

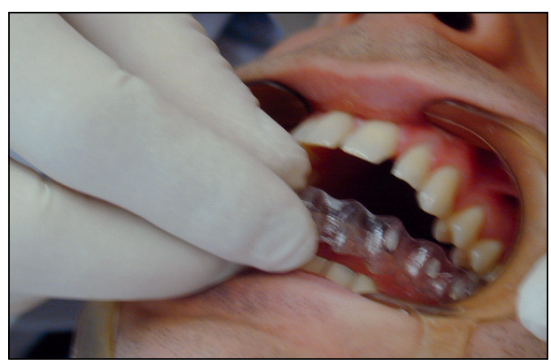

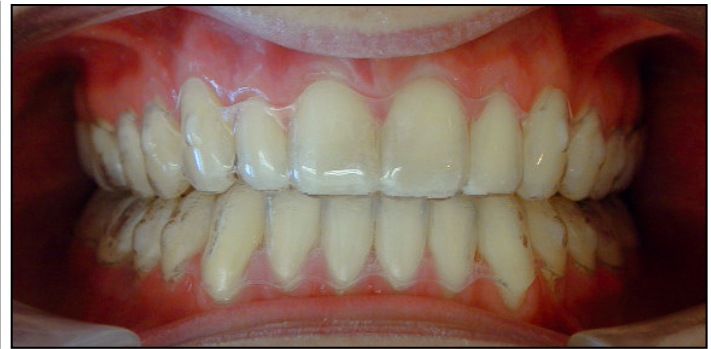

Figure 39

Phases de traitement. (a) Collage indirect des attachements, (b) aligneurs en bouche. 


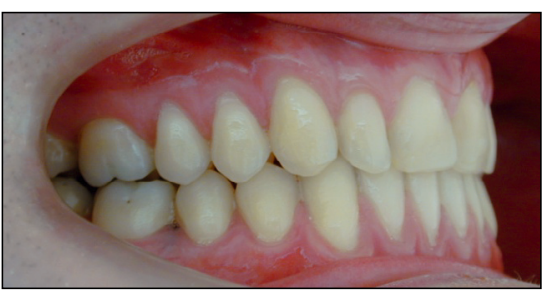

a

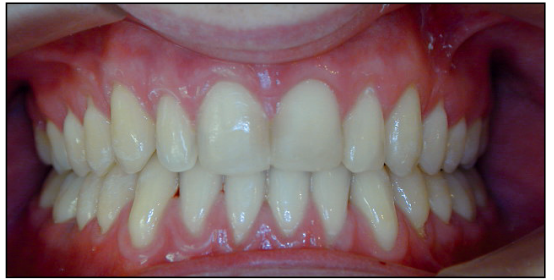

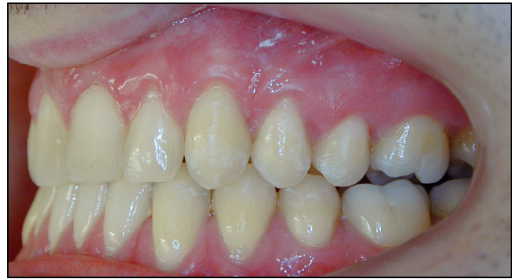

C

Figure 40

Occlusion en fin de la série d'aligneurs.

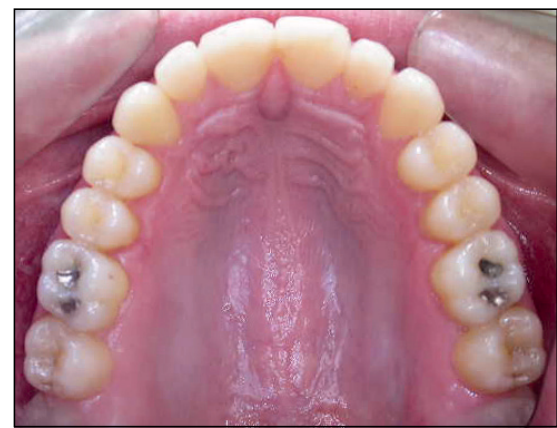

a

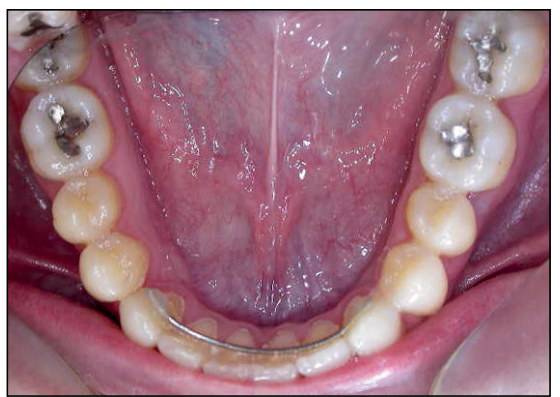

b

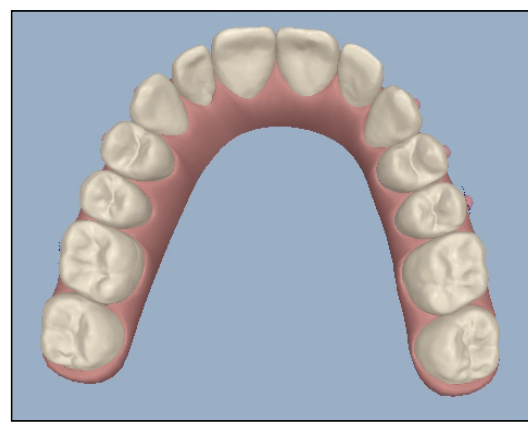

C

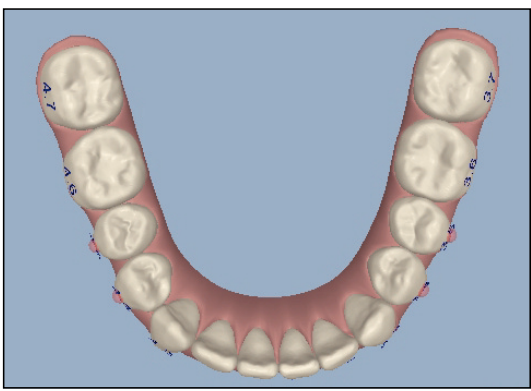

d

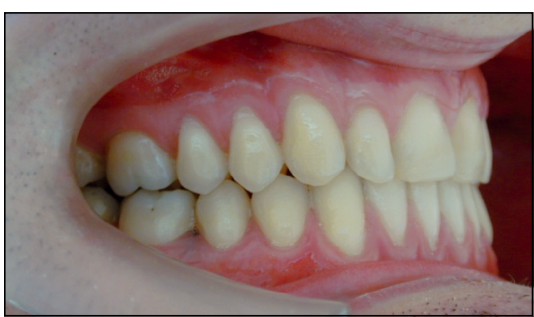

e

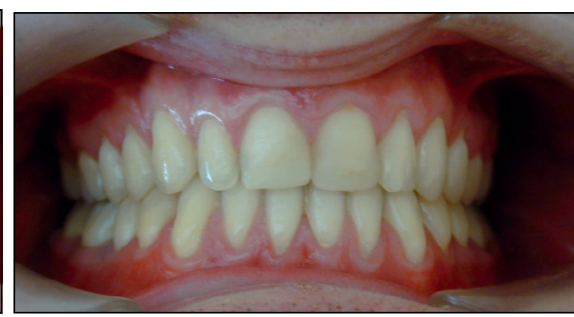

f

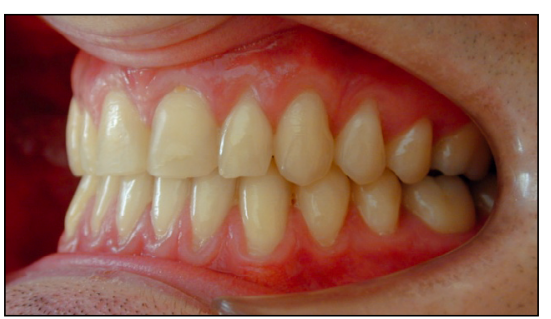

g

Figure 41

Après finitions, les secteurs latéraux sont en bonne occlusion (e à g). Les arcades terminales $(a, b)$ sont assez fidèles aux prévisions (c, d).

supérieure. Le sourire est maintenant beaucoup plus naturel (Fig. 43 et 44).

\section{Conclusions}

Pour beaucoup de patients, les considérations cosmétiques pendant le traitement peuvent être plus importantes que d'autres facteurs comme la durée de traitement, les conditions d'hygiène, le confort, la douleur. Plusieurs études ont montré qu'ils étaient prêts à payer plus pour être traités en méthode invisible [111].

Lorthodontie invisible se divise aujourd'hui en deux grandes familles d'appareils qui peuvent 


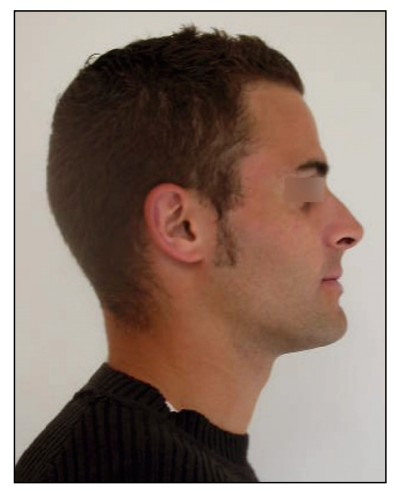

a

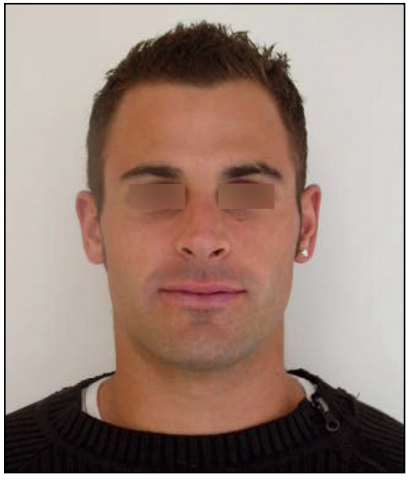

b

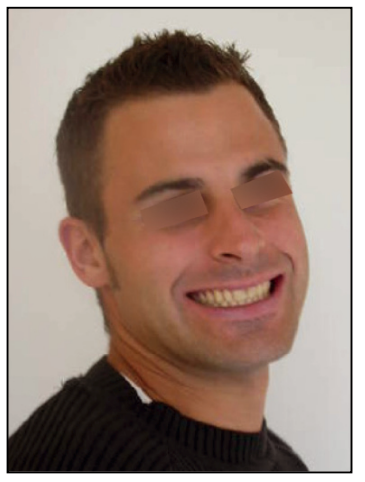

C

Figure 42

Equilibre facial conservé.

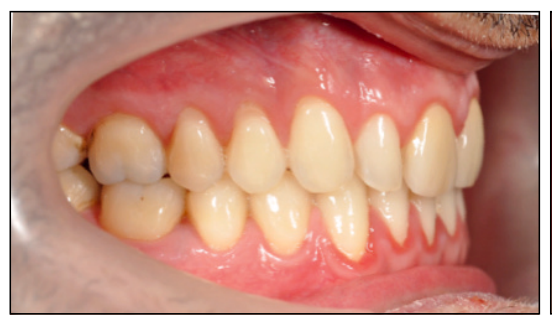

a

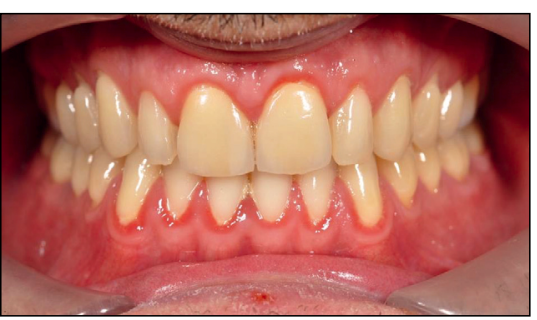

b

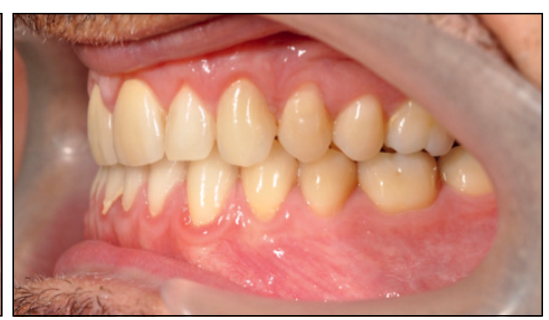

C

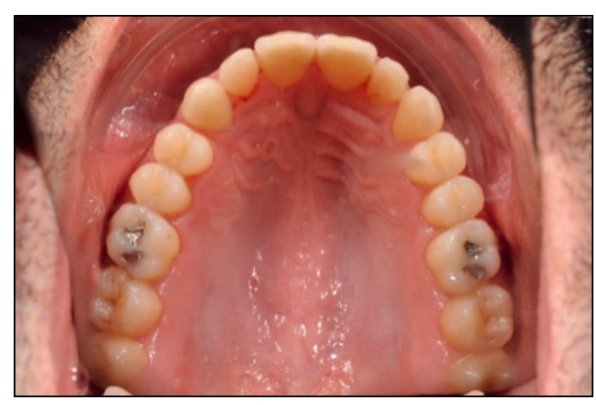

d

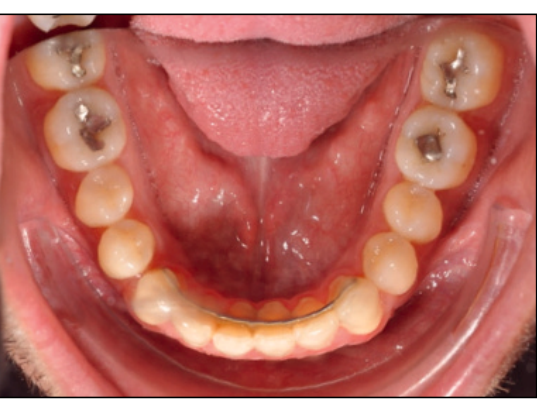

e

Figure 43

Occlusion 9 ans après, libre de contention à l'arcade supérieure.

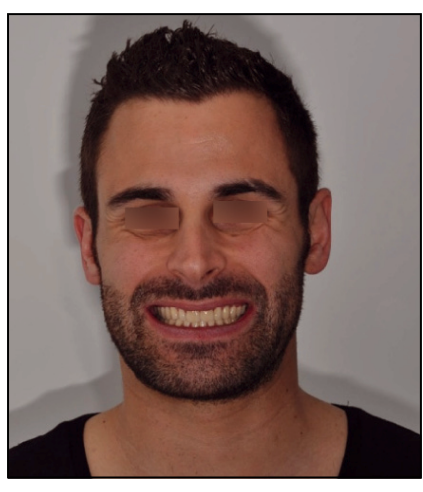

a

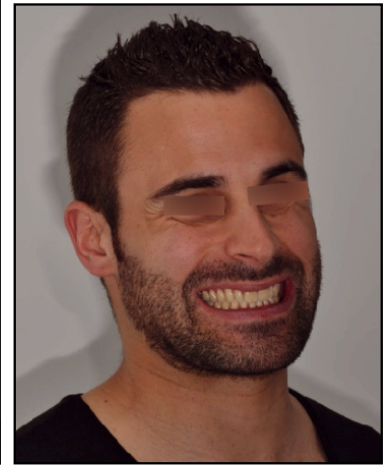

b

Figure 44

Le sourire de face et de $3 / 4$ restent harmonieux. 
produire des résultats parfaitement esthétiques aussi bien sur le dispositif thérapeutique que sur l'équilibre morphologique de la face. Pour autant, cette esthétique tant recherchée ne peut prévaloir sur les fonctions oro-faciales et l'harmonie occlusale obtenues en fin de correction. Cet équilibre fonctionnel ne doit pas non plus être dégradé en cours de traitement par les appareils employés. Pour un même résultat recherché, nous avons vu que les solutions proposées sont assez différentes sur leurs principes et leur fonctionnement modulant par là leur indication à des cas plus ou moins favorables. La littérature consacre, aussi bien pour l'orthodontie linguale que pour les systèmes d'aligneurs, des patients types remplissant des conditions idéales de traitement avec l'une ou l'autre des techniques. Elles ont en commun une possibilité de progression qui est directement liée aux progrès des technologies notamment numériques qui sont en permanence incorporées à leur fabrication. Cette logistique s'accompagne d'un coût de fabrication encore élevé. Elles s'opposent dans leur histoire où l'une s'est construite pas à pas grâce à l'opiniâtreté des cliniciens, l'autre s'étant élevée subitement par un dessein industriel volontariste. Elles diffèrent également pour les contraintes et les satisfactions qu'elles vont apporter à la fois au patient et au praticien. L'un comme l'autre doit connaître la face cachée de l'orthodontie invisible.

\section{Bibliographie}

[1] Angle EH. The latest and best in orthodontic mechanism. Dental Cosmos 1928;70:1143-1158.

[2] Angle EH. The latest and best in orthodontic mechanism. Dental Cosmos 1929;71:164-174, 260-270, 409-421.

[3] Artun J. A post treatment evaluation of multibonded lingual appliances in orthodontics. Eur J Orthod 1987;9:204-210.

[4] Baldwin DK, King G, Ramsay DS, Huang G, Bollen A M. Activation time and material stiffness of sequential removable orthodontic appliances. Part 3: premolar extraction patients. Am J Orthod Dentofacial Orthop 2008;133:837-845.

[5] Barbagallo LJ, Jones A S, Petocz P, Darendeliler MA. Physical properties of root cementum: part 10. Comparison of the effects of invisible removable thermoplastic appliances with light and heavy orthodontic forces on premolar cementum. A microcomputed-tomography study. Am J Orthod Dentofacial Orthop 2008;133:218-227.

[6] Barbagallo LJ, Shen G, Jones AS, Swain MV, Petocz P, Darendeliler MA. A novel pressure film approach for determining the force imparted by clear removable thermoplastic appliances. Annals Biomed Engin 2008;36:335-341.
[7] Baron P, Gualano C. Anchorage control performed with mini-screws and the Lingualjet Appliance. In: Echarri P, Takemoto K, et al., Nuevo Enfoque en Ortodoncia Lingual. Madrid: Ed. Ripano, 2010:15-26.

[8] Baron P, Gualano C. Brackets customisés et technique d'arc droit réunis dans le même appareil pour une orthodontie linguale simplifiée. Rev Orthop Dento Faciale 2011;45:423-436.

[9] Baron P, Gualano C. The LingualJet Appliance : a onepiece customized bracket combined with a straight wire technique. Clin Rev 2011;44-49.

[10] Baron P, Gualano G, Sempe L, Sciacca AJ, Hall G. The Manufacturing Process for LingualJet in: Romano $\mathrm{R}$, Ed. Lingual and esthetic orthodontics. Chicago: Quintessence Publishing, 2011:51-64.

[11] Becker G, Becker A. Incisor position control in lingual orthodontics. Intern Orthod 2009;7:71-95.

[12] Becker G. Cas de titularisation. Intern Orthod 2011;9:339-350.

[13] Bergström K, Halling A, Wilde B. Orthodontic care from the patients' perspective: perceptions of 27-year-old. Eur J Orthod 1998;20:319-329.

[14] Bollen AM, Huang G, King G, Hujoel P, Ma T. Activation time and material stiffness of sequential removable orthodontic appliances. Part 1: Ability to complete treatment. Am J Orthod Dentofacial Orthop 2003; 124;496-501.

[15] Boyd RL, Vlaskalic V. Three-dimensional diagnosis and orthodontic treatment of complex malocclusion with the Invisalign appliance. Semin Orthod 2001;7:274-293.

[16] Boyd RL. Surgical-orthodontic treatment of two skeletal Class III patients with Invisalign and fixed appliances. J Clin Orthod 2005:39:245-258.

[17] Breece GL, Nieberg LG. Motivations for adult orthodontic treatment. J Clin Orthod 1986;20:166-171.

[18] Bressler JM, Hamamoto S, King GJ, Bollen AM. Invisalign Therapy - A Systematic Review of Lower Quality Evidence. In: Huang GJ, Richmond S, Vig KWL, Eds. Evidence - Based Orthodontics. Wiley-Blackwell Publishing, 2011

[19] Brezniak N, Wasserstein A. Root resorption following treatment with aligners. Angle Orthod 2008;78:1119-1124.

[20] Brezniak N. The clear plastic appliance: a biomechanical point of view. Angle Orthod 2008;78:381-382.

[21] Cacciafesta V, Sfondrini MF. One-appointment correction of a scissor bite with 2D lingual brackets and fiber reinforced composites. J Clin Orthod 2006;40:409-412.

[22] Cacciafesta V. New horizons in 2D lingual orthodontics. In: Romano R, Ed. Lingual and esthetic orthodontics. Chicago: Quintessence Publishing, 2011:15-29.

[23] Campbell RI, Martorelli M, Lee HS. Surface roughness visualisation for rapid prototyping models. Computer Aided Design 2002;34:717-725.

[24] Caniklioglu C, Oztuĺrk Y. Patient discomfort: a comparison between lingual and labial fixed appliances. Angle Orthod 2005;75:86-91.

[25] Caniklioglu C, Oztuírk Y. Perceptions of patients undergoing lingual orthodontic treatment. Int Orthod 2005;3:57-63 
[26] Caniklioglu C, Oztuĺrk Y . Lingual orthodontics: treatment of non extraction case. Int Orthod 2005;3:76-86.

[27] Chenin DA, Trosien AH, Fong PF, Miller RA, Lee RS. Orthodontic treatment with a series of removable appliances. J Am Dent Assoc 2003;134:1232-1239.

[28] Cho NC, Park YC, Jo YM, Lee KJ. Combined use of miniscrews and clear appliances for the treatment of bialveolar protrusion without conventional brackets. Am J Orthod Dentofacial Orthop 2009;135:671-681.

[29] Clements KM, Bollen AM, Huang G, King G, Hujoel $\mathrm{P}, \mathrm{Ma} \mathrm{T}$. Activation time and material stiffness of sequential removable orthodontic appliances. Part 2: dental improvements. Am J Orthod Dentofacial Orthop 2003;124:502-508.

[30] Cohen-Levy J, Cohen N . Computerized analysis of occlusal contacts after lingual orthodontic treatment in adults. Int Orthod 2011;9:410-431.

[31] Kuncio D, Maganzini A, Shelton C, Freem K. Invisalign and traditional orthodontic treatment postretention outcomes compared using the American Board of Orthodontics objectif grading system. Angle Orthod 2007;77:864-869.

[32] Djeu G, Shelton C, Maganzini A. Outcome assessment of Invisalign and traditional orthodontic treatment compared with the American Board of Orthodontics objective grading system. Am J Orthod Dentofac Orthop 2005; 128:292-298.

[33] Doll GM, Zentner A, Klages U, Sergl HG. Relationship between patient discomfort, appliance acceptance and compliance in orthodontic therapy. J Orofac Orthop 2000;61:398-413.

[34] Eliades T, Bourauel C. Intraoral aging of orthodontic materials: the picture we miss and its clinical relevance. Am J Orthod Dentofacial Orthop 2005;127:403-412.

[35] Eliades T, Pratsinis H, Athanasiou AE, Eliades G, Kletsas D. Cytotoxicity and estrogenicity of Invisalign appliances. Am J Orthod Dentofacial Orthop 2009;136:100_ 103.

[36] Feder BJ. Orthodontics via Silicon Valley; a start-up uses computer modeling and venture capital to reach patients. New York Times, August 18, 2000.

[37] Fillion D. Clinical advantages of the Orapix-straight wire lingual technique. Int Orthod 2010;8:125-151.

[38] Fillion D. Computer generated conception and fabrication of transfer trays for indirect bonding of lingual attachments: The Orapix system. Rev Orthop Dento Faciale 2007:41:61-75.

[39] Fillion D. Improving patient comfort with lingual brackets. J Clin Orthod 1997;31:689-694.

[40] Fillion D. Orthodontie linguale : réflexions cliniques. Rev Orthop Dento Faciale 1990;24:475-498.

[41] Fritz U, Diedrich P, Wiechmann D. Lingual technique - Patients' characteristics, motivation and acceptance. J Orofac Orthop 2002;63:227-233.

[42] Fujita K. New orthodontic treatment with lingual bracket mushroom arch wire appliance. Am J Orthod 1979;76:657-675.

[43] Fukawa. Lingual orthodontics in the new era treatment according to criteria for occlusion and aesthetics. Int Orthod 2009; 7:370-402.
[44] Galletti C, Flauquet-Roure, Raybaud P. Treatment of class III malocclusions in adults using the Incognito lingual technique. Int Orthod 2010;8:227-252.

[45] Galletti C, Mujagic M, Simon JS. Orthodontie linguale : positionnement du premier arc dans la zone antérieure. Int Orthod 2007;5:301-326.

[46] Geron S. The lingual bracket jig. J Clin Orthod 1999;33:457-463.

[47] Geron S, Romano R, Brosh T. Vertical forces in labial and lingual orthodontics applied on maxillary incisors - a theoretical approach. Angle Orthod 2004;74:195-201.

[48] Geron S. Finishing with lingual appliance - problems and solutions. Semin Orthod 2006;12:191-202.

[49] Giancotti A, Greco M, Mampieri G. Extraction treatment using Invisalign technique. Prog Orthod 2006;7:32-43.

[50] Gorman JC, Hilgers JJ, Smith JR. Lingual orthodontics: status report Part 4, Diagnosis and treatment planning. J Clin Orthod 1983;17:26-35.

[51] Gorman JC, Smith JR. Comparison of treatment effects with labial and lingual fixed appliances. Am J Orthod Dentofacial Orthop 1991:99:202-209.

[52] Hahn W, Dathe H, Fialka-Fricke J, Fricke-Zech S, Zapf A, Kubein-Meesenburg D, et al. Influence of thermoplastic appliances' thickness on the magnitude of force delivered to an upper central incisor during tipping. Am J Orthod Dentofacial Orthop 2009;136:12.el-12.e7.

[53] Hahn W, Dathe H, Fialka-Fricke J, Fricke-Zech S, Zapf A, Kubein-Meesenburg D, et al. Torquing an upper central incisor with aligners - acting forces and biomechanical principles. Eur J Orthod 2010;32:607-613.

[54] Hiro T. Presentation of eight lingual orthodontic cases submitted for the European Board of Orthodontists examination. Int Orthod 2008;6:53-132.

[55] Hiro T. Lingual orthodontic cases treated with original Hiro brackets. in: Romano R, Ed. Lingual and esthetic orthodontics. Chicago: Quintessence Publishing, 2011:95-124.

[56] Hiro T. Preparation for new generation of lingual orthodontics: Six keys to success with lingual straight wire appliance. J Lingual Orthod 2002;2:29-47.

[57] Hohoff A, Stamm T, Ehmer U. Comparison of the effect on oral discomfort of two positioning techniques with lingual brackets. Angle Orthod 2004;74:226-233.

[58] Hohoff A, Stamm T, Goder G, Sauerland C, Ehmer U, Seifert E. Comparison of 3 bonded lingual appliances by auditive analysis and subjective assessment. Am J Orthod Dentofacial Orthop 2003;124:737-745.

[59] Hohoff A, Wiechmann D, Fillion D, Stamm T, Lippold C, Ehmer U. Evaluation of the parameters underlying the decision by adult patients to opt for lingual therapy: an international comparison. J Orofac Orthop 2003;64:135-144

[60] Hong RK, Soh BC: Customized indirect bonding method for lingual orthodontics. J Clin Orthod 1996;30:650-652.

[61] Hong RK, Sohn HW: Update on the Fujita lingual bracket. J Clin Orthod 1999;33:136-142.

[62] Honn M, Goz G. A premolar extraction case using the Invisalign system. J Orofac Orthop 2006;67:385-394. 
[63] Inami T. The treatment of class II malocclusions, combined with severe crowding and bimaxillary protrusion using a multi-lingual bracket appliance. J Jpn Assoc Adult Orthod 1996;3:76-96.

[64] Jeremiah HG, Bister D, Newton JT. Social perceptions of adults wearing orthodontic appliances: a cross-sectional study. Eur J Orthod 2011;33:476-482.

[65] Jorge JH, Giampaolo ET, Machado AL, Vergani CE. Cytotoxicity of denture base acrylic resins: a literature review. J Prosthet Dent 2003;90:190-193.

[66] Karkhanechi M, Chow D, Sipkin J, Sherman D,BoylanRJ, Normanf RG, Craig RG, Cisneros GJ. Periodontal status of adult patients treated with fixed buccal appliances and removable aligners over one year of active orthodontic therapy. Angle Orthod 2013;83:146-151.

[67] Kassas W, Al-Jewair T,Preston CB, Tabbaa S. Assessment of Invisalign treatment outcomes using the ABO Model Grading System. J World Fed Orthod 2013;2:e61-e64.

[68] Kesling HD. The philosophy of the tooth positioning appliance. Am J Orthod 1945;31:297-304.

[69] Khattab TZ, Farah H, Al-Sabbagh R, Hajeer MY, HajHamed Y. Speech performance and oral impairments with lingual and labial orthodontic appliances in the first stage of fixed treatment. A randomized controlled trial. Angle Orthod 2013;83:519-526.

[70] Kravitz ND, Kusnoto B, Agran B, Viana G. Influence of attachments and interproximal reduction on the accuracy of canine rotation with invisalign. Angle Orthod 2008;78:682-687.

[71] Kravitz ND, Kusnoto B, BeGole E, Obrez A, Agran B. How well does Invisalign work? A prospective clinical study evaluating the efficacy of tooth movement with Invisalign. Am J Orthod Dentofacial Orthod 2009; 135:27-35.

[72] Kuo E, Miller RJ. Automated custom-manufacturing technology in orthodontics. Am J Orthod Dentofacial Orthop 2003;123:578-581.

[73] Kwon JS, Lee YK, Lim BS, Lim YK. Force delivery properties of thermoplastic orthodontic materials. Am J Orthod Dentofacial Orthop 2008; 133:228-234.

[74] Kyung HM, Kim BC, Sung JH. The effect of resin base thickness on shear bonding strength in lingual tooth surface. J Clin Orthod 2002;36:320-328.

[75] Kyung HM, Park HS, Bae SM, Sung JH, Kim IB. The lingual plain-wire system with micro-implant anchorage. J Clin Orthod 2004;38:388-395.

[76] La Rocca MI, Bilello G, Caradonna C, Matranga D, Fregapane A, Curro G, Messina P. Ortodonzia estetica: vantaggi e liliti della tecnica Invisalign. Dental Cadmos 2013;81:453-461.

[77] Lagravère MO, Flores-Mir C. The treatment effects of Invisalign orthodontic aligners: a systematic review. J Am Dent Assoc 2005;136:1724-1729.

[78] Lew KK. Customized template for prefabricating initial lingual archwires. J Clin Orthod 1993;27:426.

[79] Lombardo L. STb: The light lingual system. In: Romano R, Ed. Lingual and esthetic orthodontics. Chicago: Quintessence Publishing, 2011:76-86.
[80] Long H, Zhou Y, Pyakurel U, Liao 1, Jian F, Xue J, et al. Comparison of adverse effects between lingual and labial orthodontic treatment: A systematic review. Angle Orthod 2013;83:1066-1073.

[81] Macchi A, Tagliabue A, Levrini L, Trezzi G. Philippe selfligating lingual brackets. J Clin Orthod 2002;36:42-45.

[82] Malik OH, McMullin A, Waring DT. Invisible orthodontics Part 1: Invisalign. Dent Update 2013;40:203-215.

[83] Martorelli M, Gerbino S, Giudice M, Ausiello P. A comparison between customized clear and removable orthodontic appliances manufactured using RP and CNC techniques. Dent Materials 2013;29:el-e10

[84] McMullin A, Waring DT, Malik OH. Invisible orthodontics Part 2: Lingual appliance treatment. Dent Update 2013;40:391-402.

[85] Meier B, Wiemer KB, Miethke R. Invisalign patient profiling. Analysis of a prospective survey. J Orofac Orthop 2003;64:352-358.

[86] Miethke RR, Brauner K. A comparison of the periodontal health of patients during treatment with the Invisalign system and with fixed lingual appliances. J Orofac Orthop 2007;68:223-231.

[87] Miethke RR, Vogt S. A comparison of the periodontal health of patients during treatment with the Invisalign system and with fixed orthodontic appliance. J Orofac Orthop 2005;66:219-229.

[88] Miller KB, McGorray SP, Womack R, Quintero JC, Perelmuter M, Gibson J, et al. A comparison of treatment impacts between Invisalign aligner and fixed appliance therapy during the first week of treatment. Am J Orthod Dentofacial Orthop 2007;131:302.e1-9.

[89] Miller RJ, Duong TT, Derakhshan M. Lower incisor extraction treatment with the Invisalign system. J Clin Orthod 2002;36:95-102.

[90] Miller RJ, Kuo E, Choi W. Validation of Align Technology's Treat III digital model superimposition and its case application. Orthod Craniofac Res 2003;6:143-149.

[91] Miyawaki S, Yasuhara M, Koh Y. Discomfort caused by bonded lingual orthodontic appliances in adult patients as examined by a retrospective questionnaire. Am J Orthod Dentofacial Orthop 1999;114:83-88.

[92] Morishima S, Miyaji M. Laboratory procedures of Custum Lingual Application setup system. It's problem and solution. J Jpn Lingual Orthod 1994;5:31-32.

[93] Mujagic M, Fauquet C, Galleti C, Wiechmann D, Mah J. Digital design and manufacturing of the Lingualcare bracket system. J Clin Orthod 2005;39:375-382.

[94] Muller C. Cas de titularisation. Int Orthod 2012;10:211-221.

[95] Navarro CF, Navarro MA, Perez-Salmeron J, Buccio A, Huge S. Development of the In-Ovation L bracket from GAC. LingualNews.com 2006;4(2).

[96] Nedwed V, Miethke RR. Motivation, acceptance and problems of invisalign patients. J Orofac Orthop 2005;66:162-173.

[97] Newman AA. Orthodontists market to adults seeking prettier smiles. New York Times, February 1, 2012. 
[98] Nguyen CV, Chen J. Chapter 14. In: Tuncay OC, Ed. The Invisalign system. New Malden, United Kingdom: Quintessence Publishing Company, Ltd, 2006:12-32.

[99] Nicolas S, Joseph G. Orthodontie linguale : le choix du système. Rev Orthop Dento Faciale 2007;41:9-24.

[100] Norris RA, Brandt DJ, Crawford CH, Fallah M. Restorative and Invisalign: a new approach. J Esthet Restor Dent 2002;14:217-224.

[101] Pernier C, Diemunsch C. Orthodontie « invisible». Orthod Fr 2011;82:121-144.

[102] Premaraj T, Simet S, Beatty M, Premaraj S. Oral epithelial cell reaction after exposure to Invisalign plastic material. Am J Orthod Dentofacial Orthop 2014;145:64-71.

[103] Rosvall MD, Fields HW, Ziuchkovski J, Rosenstiel SF, Johnston WM. Attractiveness, acceptability, and value of orthodontic appliances. Am J Orthod Dentofacial Orthop 2009;135:276.el-e12.

[104] Rummel V, Wiechmann D, Sachdeva RC. Precision finishing in lingual orthodontics. J Clin Orthod 1999;33:101-113.

[105] Ryokawa H, Miyazaki Y, Fujishima A, Miyazaki T, Maki $\mathrm{K}$. The mechanical properties of dental thermoplastic materials in a simulated intraoral environment. Orthod Waves 2006;65:64-72.

[106] Sattler NA. Bonding and rebonding; A small problem with a big effect in lingualtechnique. Intern Orthod 2005:3:329-337.

[107] Schuster S, Eliades G, Zinelis S, Eliades T, Bradley TG. Structural conformation and leaching from in vitro aged and retrieved Invisalign appliances. Am J Dentofacial Orthop 2004;126:725-728.

[108] Scuzzo G, Takemoto K, Mostardi G. Approche simplifiée de l'orthodontie linguale. Attache STb light lingual system. Rev Orthop Dento Faciale 2007;41:27-36.

[109] Scuzzo G, Takemoto K, Takemoto Y, Takemoto A, Lombardo L. A new lingual straight-wire technique. J Clin Orthod 2010;44:114-123.

[110] Scuzzo G, Takemoto K. Invisible orthodontics. Berlin, Quintessence, 2003; Chapter 4:39-45.

[111] Shalish M, Cooper-Kazaz R, Ivgi I, Canetti L, Tsur B, Bachar E, et al. Adult patients' adjustability to orthodontic appliances. Part I: a comparison between Labial, Lingual, and Invisalign $^{\text {TM }}$. Eur J Orthod 2012;34:724-730.

[112] Sinclair PM, Cannito MF, Goates LJ, Solomos LF, Alexander CM. Patient responses to lingual appliances. J Clin Orthod 1986;20:396-404.

[113] Smith JR, Gorman JC, Kurz C, Dunn RM. Keys to success in lingual therapy. Part 1. Clin Orthod 1986;20:252-261.

[114] Smith JR, Gorman JC, Kurz C, Dunn RM. Keys to success in lingual therapy. Part 2. Clin Orthod 1986;20:330-340.

[115] Sombuntham NP, Songwattana S, Atthakorn P, Jungudomjaroen S, Panyarachun B. Early tooth movement with a clear plastic appliance in rats. Am J Orthod Dentofacial Orthop 2009;136:75-82.

[116] Sorel O, Mehdi S, Mano MC. Procédure de collage indirect. Orthod Fr 2009;80:225-231.
[117] Sorel O. Creation of the magic bracket. Rev Orthop Dento Fac 2007;41:39-58.

[118] Takemoto K, Scuzzo G, Lombardo L, Takemoto Y. Lingual straight wire method. Int Orthod 2009;7:335-353.

[119] Takemoto K, Scuzzo G. The straight-wire concept in lingual orthodontics. J Clin Orthod 2001;35:46-52.

[120] Takemoto K, Scuzzo, Lombardo L, Takemoto Y. Lingual straight wire method. Intern Orthod 2009;7:335-353.

[121] Turpin DL. Clinical trials needed to answer questions about Invisalign. Am J Orthod Dentofacial Orthop 2005;127:157-158.

[122] Vardimon AD, Robbins D Brosh T. In-vivo von Mises strains during Invisalign treatment. Am J Orthod Dentofacial Orthop 2010;138:399-409.

[123] Vincent S. Evaluation of invisalign treatment utilizing the American board of orthodontics objective grading system for dental casts. Am J Dentofacial Orthop 2005; 127:268-269

[124] Vlaskalic V, Boyd R. Orthodontic treatment of a mildly crowded malocclusion using the Invisalign system. Aust Orthod J 2001;17:41-46.

[125] Walton DK, Fields HW, Johnston WM, Rosenstiel SF, Firestone AR, Christensen JC. Orthodontic appliance preferences of children and adolescents. Am J Orthod Dentofacial Orthop 2010;138:698.e1-e12.

[126] Warunek SP, Sorensen SE, Cunat JJ, Green LJ. Physical and mechanical properties of elastomers in orthodontic positioners. Am J Orthod Dentofac Orthop 1989;95:388-400.

[127] Wiechmann D, Gerss J, Stamm T, Hohoff A. Prediction of oral discomfort and dysfunction in lingual orthodontics: a preliminary report. Am J Orthod Dentofac Orthop 2008;133:359-364.

[128] Wiechmann D. A new bracket system for lingual orthodontic treatment. Part 2: First clinical experiences and further development. J Orofac Orthop 2003;64:372388.

[129] Wiechmann D. The new Top bracket: a step forward in lingual orthodontics? Int Orthod 2003;1:119-138.

[130] Womack WR, Day RH. Surgical-orthodontic treatment using the Invisalign system. J Clin Orthod 2008;42:237e45.

[131] Womack WR. Four-premolar extraction treatment with Invisalign. J Clin Orthod 2006;40:493-500.

[132] Wong BH. Invisalign A to Z. Am J Orthod Dentofac Orthop 2002;121:540-541.

[133] Wu A, McGrath C, Wong RW, Wiechmann D, Rabie AB. Comparison of oral impacts experienced by patients treated with labial or customized lingual fixed orthodontic appliances. Am J Orthod Dentofac Orthop 2011;139:784-790.

[134] Wu AK, McGrath C, Wong RW, Wiechmann D, Rabie AB. A comparison of pain experienced by patients treated with labial and lingual orthodontic appliances. Eur J Orthod 2010;32:403-407.

[135] Ziuchkovski JP, Fields HW, Johnston W, Lindsey D. Assessment of perceived orthodontic appliance attractiveness. Am J Orthod Dentofac Orthop 2008;133:S68-S78. 